\title{
Datando el final del Paleolítico medio en la Península lbérica. Problemas metodológicos y límites de la interpretación
}

\author{
Dating the end of the Middle Palaeolithic in the Iberian Peninsula. Methodological \\ problems and limits of interpretation
}

\author{
David Santamaría Álvarez (*) \\ Marco de la Rasilla Vives (*)
}

\section{RESUMEN}

Este artículo examina, primero, algunos problemas metodológicos relacionados con la datación absoluta/numérica de los niveles arqueológicos (fiabilidad del radiocarbono, contaminación de las muestras...), con especial énfasis en la transición del Paleolítico medio al superior ( $c a$. 40.00030.000 años BP), y se evalúan los efectos potenciales de la contaminación con carbono moderno de las muestras arqueológicas. Como corolario se exponen algunos requisitos 'estratigráficos/químicos' que debe cumplir cualquier nivel 'datado/datación', a fin de minimizar la incertidumbre que supone evaluar la fiabilidad de una fecha absoluta o numérica cuya edad real se desconoce. En segundo lugar, se analizan desde un punto de vista estratigráfico, tecnotipológico y cronológico tres yacimientos puestos como aval de una pervivencia musteriense/neandertal en el norte y sur de la Península Ibérica (El Esquilleu y Gorham's Cave) y de un intercambio genético (hibridación) entre las poblaciones sapiens y neandertales (La Sima de las Palomas). Para terminar, se discute la validez de estos modelos, basados en la cronología absoluta/numérica, y se propone un nuevo escenario asentado en la cronología relativa, y caracterizado por la no convivencia-coexistencia de las poblaciones musterienses y auriñacienses en la Península Ibérica.

\begin{abstract}
We first review some methodological problems related to the absolute/numeric dating of archaeological levels (reliability of radiocarbon dating, sample con-
\end{abstract}

(*) Área de Prehistoria, Dpto. de Historia, Facultad de Filología y Letras, Universidad de Oviedo. C/ Teniente Alfonso Martínez s/n. 33011 Oviedo. España.

Correos e.: santamariadavid@uniovi.es; mrasilla@uniovi.es Recibido 15-VI-2012; aceptado 10-X-2012. tamination...) with special emphasis on the transition from Middle to Upper Palaeolithic (ca. 40.000-30.000 years $B P)$. Furthermore we try to determine the potential effects of modern carbon contamination on the archaeological samples. As a corollary, we set out some stratigraphic/'chemical' requirements for any archaeological level/'dating' in order to minimize the uncertainty of assessing the reliability of an absolute date whose true age is unknown. Second, we analyze (from stratigraphic, typological and chronological points of view) three sites regarded as the last survival of Neanderthal/Mousterian in the north and south of the Iberian Peninsula (El Esquilleu and Gorham's Cave) and also as a gene flow (hybridization) between sapiens and neanderthals (La Sima de las Palomas). Finally, we discuss the validity of these models, based on the absolute/numeric dating, and we propose a new scenario based in the relative dating characterized by the non-coexistence of Mousterian and Aurignacian populations in the Iberian Peninsula.

Palabras clave: Paleolítico medio; Pleistoceno superior; Transición del Paleolítico medio al superior; Datación de radiocarbono; Método de pretratamiento de radiocarbono; Homo neanderthalensis; Europa occidental.

Key words: Middle Palaeolithic; Upper Pleistocene; Middle to Upper Palaeolithic transition; Radiocarbon dating; Radiocarbon Pretreatment method; Homo neanderthalensis; Western Europe.

\section{INTRODUCCIÓN (1)}

La transición del Paleolítico medio al superior en la Península Ibérica (en adelante, transición

(1) Las fechas de radiocarbono se expresan sin calibrar, excepto cuando se comparan con las dataciones obtenidas con otros métodos de datación. Están calibradas con el programa CALPAL (Weninger y Jöris 2008, curva de calibración CalPal2007-Hulu) y con la curva de calibración Intcal09 (Reimer et al. 2009). 
PM-PS, Fig. 1) constituye, en muchos sentidos (antropología, tecnologías lítica y ósea, simbolismo y, en menor medida, estrategias de subsistencia y explotación del territorio), un punto de inflexión en la historia de la humanidad y en la evolución cultural del Paleolítico. Grosso modo entre $c a .40 .000$ y 30.000 años BP tiene lugar la eclosión de las primeras culturas o complejos industriales del PS, como el Chatelperroniense, el Protoauriñaciense o el Auriñaciense, la aparición de los primeros representantes de Homo sapiens en este territorio, las posibles interacciones culturales, antropológicas y/o genéticas (hibridación, intercambios, aculturación...) entre las poblaciones indígenas (los neandertales) y las africanas (los sapiens) y, finalmente, la desaparición del Homo neanderthalensis (cuándo, dónde, cómo y por qué).

Casi todos los modelos transicionales propuestos hasta la fecha, como la 'Frontera del Ebro' (Zilhão 1993, 2000, 2006), la existencia de refugios neandertales en el norte y sur de la Penín-

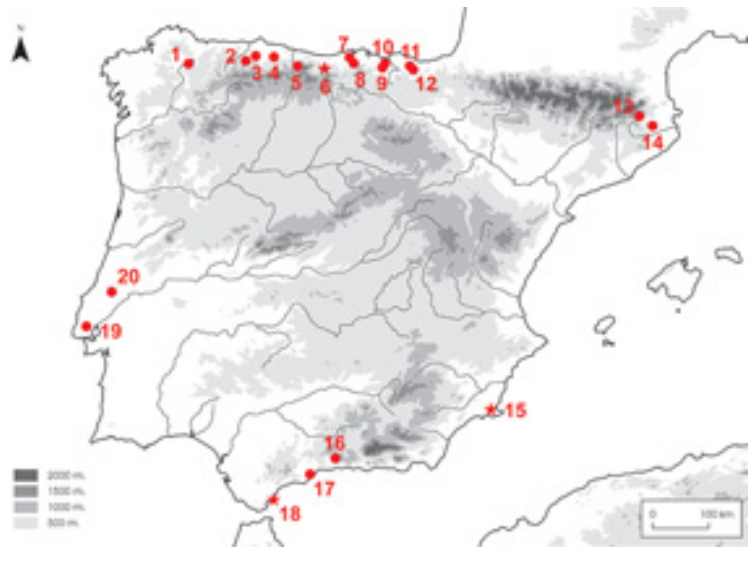

Fig. 1. Mapa de la Península Ibérica con indicación de los yacimientos citados en el texto ( $\star$ los principales). 1. A Valiña (Castroverde, Lugo), 2. El Conde (Santo Adriano, Asturias), 3. La Viña (Oviedo, Asturias), 4. El Sidrón (Piloña, Asturias), 5. La Güelga (Cangas de Onís, Asturias), 6. El Esquilleu (Cillorigo de Liébana, Cantabria), 7. El Pendo (Camargo, Cantabria), 8. Morín (Villaescusa, Cantabria), 9. Polvorín (Karrantza, Vizcaya), 10. Venta Laperra (Karrantza, Vizcaya), 11. Ekain (Deba, Guipúzcoa), 12. Labeko koba (Arrasate, Guipúzcoa), 13. Els Ermitons (Garrotxa, Girona), 14. L'Arbreda (Serinya, Girona), 15. La Sima de las Palomas (Torre Pacheco, Murcia), 16. Zafarraya (Alcaucín, Malaga), 17. Bajondillo (Torremolinos, Málaga), 18. Gorham's Cave (Gibraltar, Reino Unido), 19. Pego do Diabo (Loures, Portugal), 20. Oliveira (Almonda, Portugal). sula Ibérica (PI) (Baena et al. 2005; Baena et al. 2006; Finlayson et al. 2006; Baena et al. 2012) o el Auriñaciense de Transición (Cabrera et al. 1993; Cabrera et al. 2001), se asientan en mayor o menor medida en una evaluación parcial, y en ocasiones interesada, del conjunto de dataciones, en su mayoría de radiocarbono, disponibles para este periodo. La interpretación y evaluación de estas fechas está condicionada al menos por tres factores: a) las técnicas de pretratamiento utilizadas en los laboratorios (Longin, Ultrafiltración, Ninhydrina, ABA, ABOx...) y el límite de aplicabilidad del radiocarbono (variable de un laboratorio a otro, aunque inferior a $\sim 60 / 50.000$ años $\mathrm{BP}$ ); b) el tipo de muestra: hueso, concha o carbón, entre otras; c) el contexto estratigráfico y cultural de la muestra.

Estos factores han sido ampliamente tratados (Zilhão y d'Errico 1999, 2000, 2003; Pettitt y Bailey 2000; Jöris et al. 2003; Bronk Ramsey et al. 2004a; Bronk Ramsey et al. 2004b; Higham et al. 2006, 2009; Brock et al. 2007; Adler y Jöris 2008; Blockley et al. 2008; Jöris y Street 2008; Weninger y Jöris 2008; Jöris et al. 2011; Higham 2011) y forman parte del debate sobre el origen y el desarrollo de las primeras industrias del PS, la perduración del Musteriense, y por ende sobre la llegada del Homo sapiens al continente europeo y la extinción de los últimos neandertales (Fortea et al. 2008).

\section{LOS EFECTOS DE LA CONTAMINACIÓN CON CARBONO MODERNO: UN MODELO TEÓRICO A PARTIR DE LA CURVA DE DECAIMIENTO DEL C14}

La contaminación de una muestra arqueológica puede tener un origen natural y/o antrópico. Los contaminantes naturales más citados son la intrusión de raíces en los niveles arqueológicos y la circulación de ácidos húmicos procedentes de la descomposición de los vegetales (Hedges y van Klinken 1992; van Klinken 1999). La contaminación antrópica suele producirse durante la manipulación y el tratamiento de la muestra (desde su recogida en el yacimiento hasta su procesado en el laboratorio), teniendo efectos directos, aunque variables, en los resultados de la prueba.

Trab. Prehist., 70, N. ${ }^{\circ}$ 2, julio-diciembre 2013, pp. 241-263, ISSN: 0082-5638

doi: $10.3989 /$ tp.2013.12111 
La desintegración nuclear de los isótopos radiactivos (como el radiocarbono) se manifiesta de forma exponencial, es decir, el ritmo de decaimiento de los isótopos es proporcional al número de átomos restante. El periodo de semidesintegración, o semivida, del C14 es de 5.730 \pm 40 años (Godwin 1962; Stuiver y Polach 1977). Transcurrido cada periodo, la cantidad de C14 presente en una muestra se reduce a la mitad; así, tras una semivida (5.730 años) el contenido de $\mathrm{C} 14$ es $1 / 2$ (o el 50\%) de la cantidad inicial, después de dos periodos (11.460 años) la cantidad de C14 remanente es $1 / 4$ (o el 25\%), y así sucesivamente (Tab. 1 y Fig. 2). Esto significa que tras 7 periodos de semidesintegración (i.e. tras 40.000 años transcurridos) la cantidad remanente de radiocarbono es inferior al $1 \%(0,78 \%)$, o $1 / 128=$ 0,0078, del contenido inicial. En la actualidad, algunos laboratorios de datación proporcionan fechas próximas $\mathrm{a} \pm 60.000$ años $\mathrm{BP}$, lo que equivale a \pm 11 periodos de semidesintegración. Transcurri-

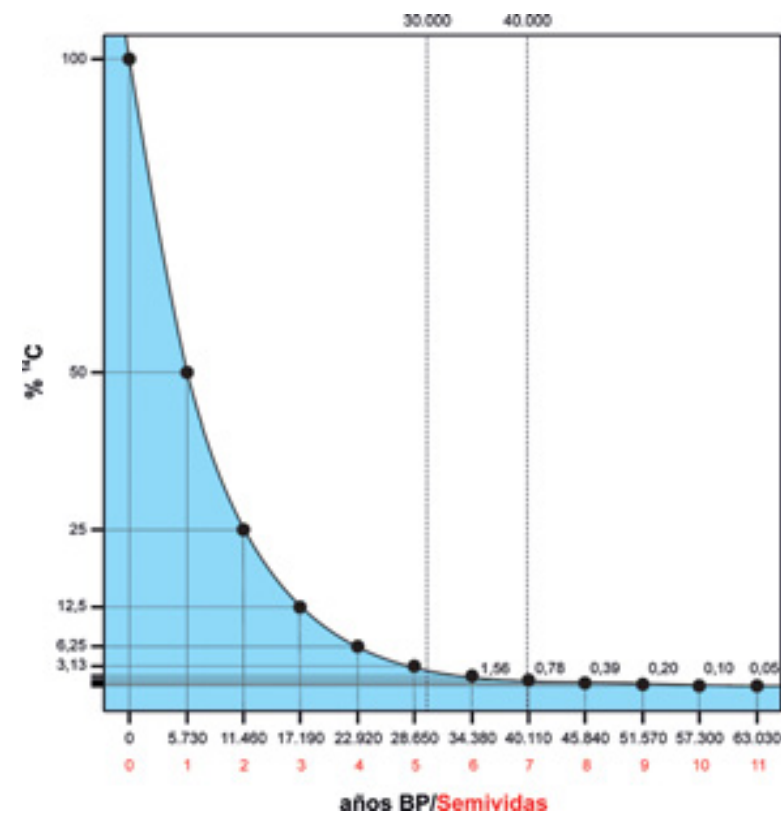

Fig. 2. Curva de decaimiento estándar del radiocarbono.

\begin{tabular}{|c|c|c|c|c|c|c|c|}
\hline \multirow{2}{*}{$\mathbf{n}$} & \multicolumn{2}{|c|}{ Decaimiento ${ }^{14} \mathrm{C}$} & \multirow{2}{*}{$\begin{array}{l}\text { Edad real } \\
\text { (años BP) }\end{array}$} & \multicolumn{4}{|c|}{ Edad contaminada (años BP) } \\
\hline & $14 \mathrm{~A}$ & $\% 14 C$ & & 0,5 & 1 & 1,5 & 2 \\
\hline 0 & 1 & 100 & 0 & - & - & - & - \\
\hline 1 & 0,5 & 50 & 5.730 & 5.648 & 5.567 & 5.486 & 5.406 \\
\hline 2 & 0,25 & 25 & 11.460 & 11.297 & 11.136 & 10.979 & 10.824 \\
\hline 3 & 0,125 & 12,5 & 17.190 & 16.867 & 16.555 & 16.254 & 15.964 \\
\hline 4 & 0,0625 & 6,25 & 22.920 & 22.285 & 21.694 & 21.143 & 20.626 \\
\hline 5 & 0,03125 & 3,125 & 28.650 & 27.424 & 26.356 & 25.410 & 24.562 \\
\hline 6 & 0,015625 & 1,5625 & 34.380 & 32.086 & 30.292 & 28.818 & 27.568 \\
\hline 7 & 0,007812 & 0,78125 & 40.110 & 36.022 & 33.299 & 31.253 & 29.615 \\
\hline 8 & 0,00390625 & 0,390625 & 45.840 & 39.029 & 35.345 & 32.806 & 30.866 \\
\hline 9 & 0,001953125 & 0,1953125 & 51.570 & 41.075 & 36.596 & 33.707 & 31.570 \\
\hline 10 & 0,000976562 & 0,0976562 & 57.300 & 42.326 & 37.301 & 34.198 & 31.947 \\
\hline 11 & 0,000488281 & 0,0488281 & 63.030 & 43.031 & 37.677 & 34.454 & 32.141 \\
\hline
\end{tabular}

Tab. 1. Ritmo de decaimiento del radiocarbono y efectos potenciales de la contaminación con carbono moderno. n: periodos transcurridos, $\mathrm{A}^{14}$ : contenido de $\mathrm{C} 14$ remanente, $\%{ }^{14} \mathrm{C}$ : porcentaje de $\mathrm{C} 14$ remanente, Niveles de contaminación: $0,5 \%, 1 \%$, $1,5 \%$ y $2 \%$. Para calcular el porcentaje de carbono moderno necesario para que una fecha determinada (a) se date en otra (b) se ha utilizado el siguiente algoritmo $\mathrm{C}=\left(A_{b}^{14}-A_{a}^{14}\right) 100$ : donde $\mathrm{C}$ es el \% de carbono 14 moderno necesario para explicar una desviación cronológica de x años, $A_{b}^{14}$ es el contenido de ${ }^{14} \mathrm{C}$ de la fecha $b$ y $A_{a}^{14}$ es el contenido de ${ }^{14} \mathrm{C}$ de la fecha $a$. El contenido de carbono $14\left(\mathrm{~A}^{14}\right)$ de una fecha es igual a: $A^{14}=\frac{1}{2^{n}}$ donde $\mathrm{n}$ es igual al número de periodos transcurridos, $n=\frac{a}{5730}$ siendo $a$ la fecha de radiocarbono.

Por ejemplo, el porcentaje de carbono 14 moderno necesario para que una fecha de 47.433 años se date en 34.866 años es igual a $1,15 \%$

$\mathrm{a}=47.433$ años $\mathrm{BP} ; \mathrm{n}_{\mathrm{a}}=8,278 ; \quad A_{a}^{14}=0,003221$

$\mathrm{b}=34.866$ años BP; $\mathrm{n}_{\mathrm{b}}=6,085 ; \quad A_{b}^{14}=0,014733$

$\mathrm{c}=1,15 \%$ 
do este tiempo, el porcentaje de $\mathrm{C} 14$ remanente es inferior al $0,05 \%$ del contenido inicial.

La curva de desintegración del radiocarbono explica por qué los efectos de la contaminación, o rejuvenecimiento de la estimación $\mathrm{C} 14$, se intensifican notablemente en las muestras más antiguas (Hedges y van Klinken 1992; Mercier y Valladas 1993; Schwarcz 1993; Jöris et al. 2003; Higham et al. 2006; Jöris y Street 2008; Higham 2011; Jöris et al. 2011). A partir de \pm 38.000 años BP la cantidad de C14 en una muestra arqueológica es inferior al 1\% del contenido inicial. Estas muestras son más sensibles a los efectos de la contaminación por el bajo contenido de C14 que conservan y por el ritmo de decaimiento del radiocarbono. En la práctica, un $1 \%$ extra de contaminantes modernos, no eliminados durante el pretratamiento de la muestra, supone un rejuvenecimiento de la datación de \pm 6.000 años. Esta situación podría crear un efecto ilusorio de contemporaneidad entre niveles arqueológicos cuya edad verdadera difiriera en varios miles de años (Jöris et al. 2003; Fortea et al. 2008). Según Schwarcz (1993: 36), in practice, C14 dates became highly subsceptible to contamination for samples older than about $35 \mathrm{ka}$.

Para evaluar los efectos potenciales de la contaminación con carbono moderno se ha elaborado un modelo matemático a partir de la curva de decaimiento estándar del C14 (Fig. 2). Las referencias son las edades y el contenido de $\mathrm{C} 14$ remanente $\left(\mathrm{A}^{14}\right)$ de los 11 primeros periodos de semidesintegración del radiocarbono (Tab. 1). Asimismo, se han establecido cuatro niveles de contaminación distribuidos entre $0,5 \%$ y $2 \%$ (a efectos de cálculo estos porcentajes se expresan como 0,005 y 0,02 ), espaciados en intervalos regulares de $0,5 \%(00,005)$. A cada nivel se suma el contenido de $\mathrm{C} 14\left(\mathrm{~A}^{14}\right)$ del periodo en cuestión. La edad contaminada de la muestra se calcula según la ecuación de decaimiento del radiocarbono (Stuiver y Polach 1977; Mook 2002):

$$
t_{c}=-8267 \ln \left(A_{c}^{14}\right)
$$

donde $t_{c}$ es la edad contaminada que queremos conocer, 8267 es la vida media del C14 calculada para un periodo estándar de 5.730 años, $A_{c}^{14}$ es el contenido de C14 remanente + el nivel de contaminación (es decir, la suma del C14 real + el nivel de contaminación estipulado a priori) y ln representa el logaritmo natural. Por ejemplo, en una muestra hipotética cuya edad real fuese 34.380 años BP la cantidad de ${ }^{14} \mathrm{C}$ remanente es igual a $1 / 64$ o $\mathrm{A}^{14}=0,015625$ (Tab. 1). Primero se suman los índices de contaminación estipulados a priori $(0,005 ; 0,01 ; 0,015$ y 0,02$)$ al contenido de C14 remanente $\left(A_{0,5}^{14}=0,020625 ; A_{1}^{14}=\right.$ 0,$025625 ; A_{1,5}^{14}=0,030625$ y $\left.A_{2}^{14}=0,035625\right)$, a continuación se calculan las edades contaminadas de la muestra según la ecuación de decaimiento del radiocarbono (1.0).

$$
\begin{aligned}
& t_{0,5}=-8267 \ln \left(A_{0,5}^{14}\right) \\
& t_{0,5}=-8267 \ln (0,020625) \\
& t_{0,5}=32.086
\end{aligned}
$$

En este caso $t_{0,5 \%}$ es la edad de la muestra con un $0,5 \%$ de contaminación. Por lo tanto, la edad contaminada (a $0,5 \%$ ) de una muestra ficticia cuya edad real fuese 34.380 años BP es igual a 32.086 años BP o si se prefiere 2.294 años más joven. Los resultados obtenidos se resumen en la tabla 1 y en la Fig. 3. En general, se observa un incremento significativo de los efectos contaminantes, o rejuvenecimiento de las fechas, a medida que disminuye la cantidad de C14.

Las muestras con más de $25 \%$ de $\mathrm{C} 14$ (es decir con $<$ de dos semividas) son inmunes a los niveles de contaminación utilizados en este es-

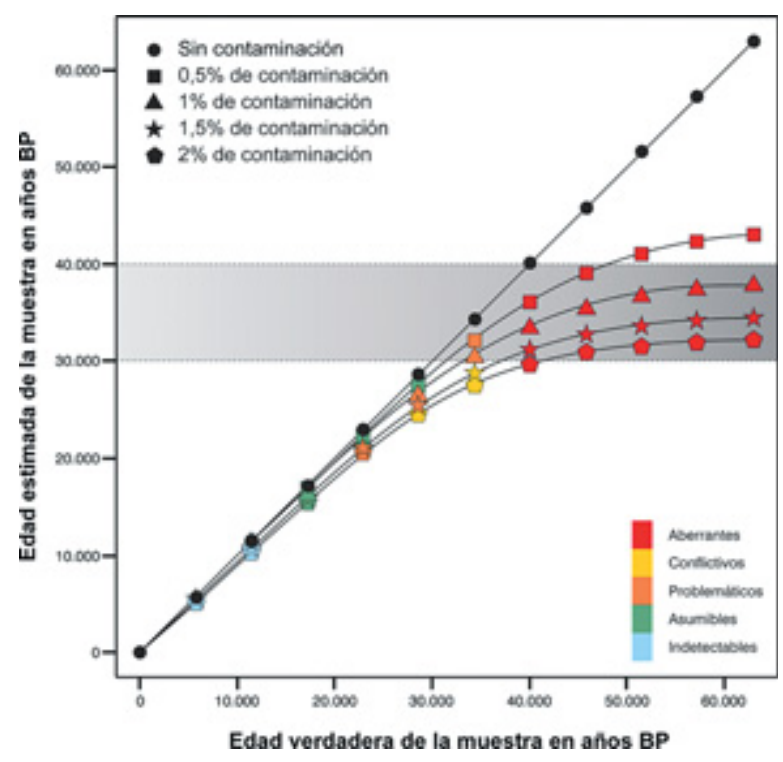

Fig. 3. Desviaciones cronológicas producidas por la contaminación de las muestras con carbono moderno. 
tudio $(0,5 \%, 1 \%, 1,5 \%$ y $2 \%)$. En estos casos, la contaminación de las muestras no provoca un rejuvenecimiento significativo de las dataciones (desviaciones cronológicas máximas de -600 años). Sus efectos pueden considerarse nulos o muy bajos, en todo caso 'indetectables'.

Las muestras que tienen entre $25 \%$ y $3,125 \%$ de C14 (es decir con más de dos semividas y menos de seis) son sensibles a niveles altos de contaminación $(1,5 \%-2 \%)$. En cambio, los efectos producidos por la contaminación pueden considerarse 'asumibles' cuando sus niveles son inferiores a 1\% (desviaciones cronológicas máximas de -1.200 años para las muestras más antiguas) y medios/medios-altos (es decir, 'conflictivos-problemáticos') cuando esos niveles igualan o superan 1\% (medios para las muestras con menos de 4 semividas y medios-altos para las muestras con más de 4 semividas).

Por último, las muestras con más de seis semividas (> 34.380 años BP) son muy sensibles a cualquier nivel de contaminación. Niveles bajos $(0,5 \%)$ provocan desviaciones cronológicas muy importantes (entre -2.000 y -20.000 años) y con niveles del $2 \%$ la edad de las muestras se rejuvenece entre -7.000 y -31.000 años. Los efectos producidos por la contaminación pueden considerarse sin lugar a dudas altos o muy altos, 'aberrantes'.

Resumiendo, el rejuvenecimiento de una fecha de radiocarbono es inversamente proporcional al contenido de $\mathrm{C} 14$ remanente, o si se prefiere directamente proporcional a la edad verdadera de la muestra (Fig. 3).

En los yacimientos de la PI con series estratigráficas largas PM-PS (La Viña, Morín, Castillo, Bajondillo, Cova Gran, Romaní o L'Arbreda) el Musteriense se localiza siempre a muro del PS (ausencia de interestratificaciones), independientemente de la situación geográfica del yacimiento (región cantábrica, mediterránea, sur de la PI). Debemos aceptar, por tanto, que los niveles musterienses, más antiguos desde un punto de vista cronoestratigráfico, son más proclives a estar contaminados que los niveles adscritos al PS (ya que han estado más tiempo expuestos) y mucho más sensibles a los efectos de la contaminación (ya que son más antiguos). Además, en la actualidad it is often impossible to establish whether the material is contaminated or not, and apart from obvious anomalous samples, it is still difficult to judge until which age LMP [Latest Middle Palaeolithic] dates may be considered reliable (Jöris et al. 2003: 19).
Como se observa en la figura 3 , el ritmo de desintegración del radiocarbono provoca un efecto barrera en torno al 40.000 BP para todas las muestras contaminadas superiores a 45.000 años (nivel de contaminación igual a $\sim 0,5 \%$, Tab. 1; Jöris y Street 2008). Con un porcentaje de contaminación igual a $1,5 \%$ todas las muestras $>45.000$ años se datarían en torno al 35.000 BP. Por último, superar la barrera del 28.000 BP implica niveles de contaminación de tan solo un 3\% para todas las muestras superiores a esa fecha (Tab. 1). Un escenario hipotético en el que las poblaciones musterienses hubiesen desaparecido antes del 45.000 BP (sin calibrar) y las primeras culturas auriñacienses se fechasen en el 35/38.000 BP (sin calibrar) quedaría totalmente desfigurado por los efectos de la contaminación, creando la ilusión de una coexistencia mustero-auriñaciense de varios miles años.

Los límites generalmente aceptados para el inicio y el fin de la transición (i.e. 40.000-30.000 años $\mathrm{BP}$ en cronología absoluta) actúan en la práctica como un muro de contención que atenúa los efectos de la contaminación (Fig. 3), creando posibles escenarios de coexistencia o convivencia musteroauriñaciense (Jöris et al. 2003; Jöris y Street 2008; Jöris et al. 2011) que, en términos cronoestratigráficos, resta por demostrar. Conviene preguntarse, por tanto, si la perduración del Homo neanderthalensis/ Musteriense en la PI hasta \pm 24.000 años BP (Finlayson et al. 2006; Finlayson et al. 2008; Baena et al. 2012) es un acontecimiento histórico/arqueológico suficientemente documentado (desde un punto de vista estratigráfico, tecno-tipológico...) o por el contrario es consecuencia de la contaminación con carbono moderno de las muestras arqueológicas. Así, el $\sim 63 \%(\mathrm{n}=143)$ de las fechas $\mathrm{C} 14$ musterienses son posteriores al 40.000 BP (solo 85 dataciones son anteriores a esta fecha, Santamaría 2012) (2). ¿Debemos concluir, por lo tanto, que la PI fue escenario de un repoblamiento musteriense posterior al $40.000 \mathrm{BP}$ ¿ ¿No es más sensato pensar que esas fechas son el resultado de la contaminación con carbono moderno de las muestras arqueológicas, o del límite de aplicabilidad específico de cada laboratorio? ¿Cómo interpretar sino el vacío de dataciones que hay antes del 45.000 BP?

Lo que es sumamente arriesgado para estos momentos es plantear escenarios multiculturales basa-

(2) Véase el material complementario de su versión electrónica. 
dos en exclusiva en la cronología absoluta (Zilhão 1993, 2000, 2006; Hublin et al. 1995; Baena et al. 2005; Maíllo 2005a; Finlayson et al. 2006; Bernaldo de Quirós et al. 2008; Jennings et al. 2009; Baena et al. 2012), a pesar de que estos autores reconocen en todo momento que sus interpretaciones están condicionadas por los límites de los métodos de datación radiométrica. No obstante, los especialistas en cronología llevan mucho tiempo advirtiendo del peligro implícito de interpretar las fechas radiométricas en términos histórico-evolutivos (Hedge y van Klinken 1992; Mercier y Valladas 1993; Pettitt y Pike 2001; Jöris y Street 2008; Jöris et al. 2011).

\section{CONSIDERACIONES A TENER EN CUENTA A LA HORA DE EVALUAR UNA FECHA DE RADIOCARBONO}

Una fecha de radiocarbono correspondiente a la transición PM-PS (ca. 30.000-40.000 años BP) no es necesariamente fiable. A lo sumo ha superado los protocolos anticontaminación del laboratorio donde se obtuvo. ¿Basta para incluirla en el debate? Nosotros creemos que la aceptación de una fecha de radiocarbono exige un análisis riguroso de ciertos parámetros estratigráficos e isotópicos que minimice la incertidumbre que supone evaluar la fiabilidad de una fecha absoluta cuya edad real se desconoce.

\subsection{Requisitos estratigráficos}

La integridad estratigráfica de un nivel arqueológico debe evaluarse desde la estratigrafía, la sedimentología, la tafonomía, la edafología y la tecnología entre otras disciplinas. La interpretación de una fecha de radiocarbono debe tener en cuenta siempre el estado de conservación del nivel, o niveles, datado/s. La proyección estratigráfica y en planta de la muestra datada es un requisito imprescindible en los yacimientos donde se hayan identificado procesos sin y/o postdeposicionales de cierta envergadura (discordancias erosivas, solifluxiones, madrigueras, pisoteo, basureros, contaminación inter-estratigráfica).

La caracterización cultural del nivel datado no es una cuestión baladí, especialmente en so- ciedades cazadoras-recolectoras con un sustrato tecno-tipológico (en ocasiones poco o nada diagnóstico) que, en muchos aspectos, se mantiene invariable durante gran parte del Paleolítico. Muchos yacimientos transicionales (desde un punto de vista cronológico) de la PI carecen de estudios tecno-tipológicos rigurosos (clasificación tipológica, cadenas operativas, remontajes inter e intra-estratigráficos, localización estratigráfica y en planta de los restos arqueológicos) que permitan contrastar la entidad cultural del nivel estudiado, y comparar los resultados con otros yacimientos asignados al mismo complejo industrial. Si manejamos la clasificación propuesta por Barandiarán et al. (1996: 279-280): conjuntos líticos "paupérrimos" ( $\leq 50$ piezas retocadas), "exiguos" (51-100 útiles), "discretos" (101-200 útiles) y "suficientes" (>201 útiles), el panorama resulta bastante desolador. Por ejemplo, el Chatelperroniense ha sido identificado en 9 niveles arqueológicos de 8 yacimientos de la PI: A Valiña, La Güelga, Pendo, Morín, Polvorín (2 niveles), Venta Laperra, Ekain y Labeko koba (Maíllo 2007; Andrés 2009). De esos 9 niveles: 6 (66,7\%) tienen menos de 50 útiles (Polvorín 4 y 5, La Güelga 1 zona D interior, Venta Laperra II, Ekain X y Labeko koba IX inferior), 2 (22,2\%) entre 50 y 100 útiles (Pendo VII y A Valiña 1) y solo Morín 10 $(11,1 \%)$ más de 200 útiles. El Chatelperroniense cantábrico se revela, pues, como un complejo industrial quimérico, pobremente representado y en general mal caracterizado. Además Morín 10, el único yacimiento "chatelperroniense" con un repertorio instrumental "suficiente", tiene serios problemas estratigráficos (contactos discordantes, erosiones, crioturbación) que afectan a los niveles musterienses (11 y 12), chatelperroniense y protoauriñacienses (Laville y Hoyos 1994), por ahora, sin evaluar desde un punto de vista tecnotipológico (i.e. contaminaciones inter-estratigráficas). A este respecto conviene recordar que las puntas de Chatelperrón aparecen tanto en el nivel 10 chatelperroniense $(n=13)$ como en los niveles 8 y 9 protoauriñacienses suprayacentes $(\mathrm{n}=9)$ (Maíllo 2002, 2005b).

El panorama es algo más favorable en relación con el Auriñaciense y el Musteriense, sin llegar a ser plenamente satisfactorio. Muchos yacimientos auriñacienses y musterienses datados en la transición PM-PS carecen de estudios tecno-tipológicos rigurosos que permitan contrastar su adscripción 
cultural. Algunos yacimientos en el meollo de ciertos modelos interpretativos (Pego do Diabo, Oliveira, Gorham's Cave, Zafarraya) tienen unas colecciones líticas y/u óseas paupérrimas o exiguas, a veces poco o nada diagnósticas. Todo ello repercute en nuestra percepción del pasado, y por ende de la transición PM-PS, sobrevalorando algunos yacimientos y complejos tecnológicos que en otros contextos pasarían desapercibidos.

La asociación de la muestra datada y del evento que se quiere datar (e.g. carbón recogido en un hogar, industria ósea, huesos humanos, marcas de corte...) es crucial donde se haya documentado una ocupación alterna de humanos y carnívoros; en yacimientos con una baja densidad de artefactos líticos/óseos (ocupaciones humanas esporádicas) o con problemas estratigráficos (vide supra). En niveles sin estas características la asociación muestra-evento es menos importante.

La coherencia estratigráfica de las fechas se basa en el principio de la superposición de los estratos de Steno: los estratos inferiores son siempre los más antiguos bajo condiciones normales de sedimentación, i.e sin erosiones, inversiones estratigráficas... De forma paralela, las dataciones procedentes de los niveles inferiores deben ser más antiguas que las de los niveles superiores. Las "inversiones cronológicas" pueden resultar de dos procesos: a) desplazamientos verticales de las muestras (o contaminaciones interestratigráficas), y b) contaminación de la muestra con carbono moderno (rejuvenecimiento de la muestra). La coherencia intraestratigráfica de las dataciones permite contrastar la fiabilidad de una datación absoluta o numérica comparándola con otras fechas cuya edad real se estima, grosso modo, contemporánea.

\subsection{Parámetros químicos}

Los indicadores elementales e isotópicos permiten evaluar la fiabilidad de una fecha de radiocarbono. Varían según el tipo de muestra (hueso, quemado o no, diente, carbón, sedimento, concha...). El hueso y el carbón constituyen más del 95\% de las muestras datadas en la PI. Muchos investigadores consideran el carbón uno de los materiales más adecuados (Bird et al. 1999; Pessenda et al. 2001; Jöris et al. 2003, Jöris y Street 2008; Higham et al. 2009). Sin embargo le afectan problemas que no pueden obviarse: a) en ocasiones no se puede justificar la asociación de la muestra datada y del evento que se quiere datar (Zilhão y d'Errico 1999), e.g. fragmentos de carbón recogidos en niveles con una baja densidad de utensilios líticos y óseos, y por lo tanto proclives a desplazamientos verticales; o b) el grado de contaminación de la muestra siempre es difícil de evaluar y cuantificar (Chapell et al. 1996).

El pretratamiento habitual utilizado en los laboratorios de datación se denomina ABA (ácidobase-ácido). Su objetivo es eliminar los carbonatos y los ácidos húmicos absorbidos durante el enterramiento de la muestra. No obstante, en ocasiones no se consiguen eliminar todos los contaminantes orgánicos de la muestra, e.g. ácidos húmicos (Chapell et al. 1996; Gillespie 1997; Bird et al. 1999; Jöris y Street 2008), comprometiendo los resultados de la prueba radiométrica. A medida que nos acercamos al límite de aplicabilidad del radiocarbono, la transición PM-PS, los efectos de la contaminación con carbono moderno (i.e. el rejuvenecimiento de la edad C14) se intensifican notablemente (vide supra), hasta el punto de obtener fechas absolutas (en general transicionales) en muestras sin C14 remanente, i.e. más allá del límite de aplicabilidad del radiocarbono (Chapell et al. 1996).

Un nuevo tratamiento (Bird et al. 1999) incorpora a la secuencia clásica (ABA) una fase adicional de oxidación: ácido-base-oxidación ( $\mathrm{ABOx})$. Este método elimina de un modo más eficiente el carbono exógeno de la muestra (los contaminantes). En ocasiones las diferencias entre ambos métodos son de $\pm 10 / 15.000$ años, ampliando a su vez el límite de aplicabilidad del radiocarbono hasta \pm 60.000 años BP (Bird et al. 1999; Brock y Higham 2009; Higham et al. 2009). Para evaluar la calidad de una muestra de carbón los indicadores químicos utilizados son un $\% \mathrm{C}$ de la muestra comprendido entre 50\% y 70\% (Braadbaart et al. 2009) y un $\delta^{13} \mathrm{C}$ (o ratio delta del carbono 13, ${ }^{13} \mathrm{C} /{ }^{12} \mathrm{C}$ ) entre $-26 \%$ y $-22 \%$.

Varias razones explican la selección de muestras de hueso para datar: a) cantidad; b) es más fácil justificar la asociación de la muestra con el evento que se quiere datar (marcas de corte, hueso humano, industria ósea) (Zilhão y d'Errico 1999). No obstante, problemas inherentes a su naturaleza comprometen la fiabilidad de las fechas (van Klinken 1999: 687): a) alteraciones diagenéticas 
que hayan degradado la estructura y la calidad del colágeno, b) la contaminación de la muestra con carbono exógeno (moderno o fósil) y c) el método de pretratamiento utilizado.

La calidad de una muestra ósea se evalúa mediante parámetros químicos como la concentración relativa de colágeno (\% yield) y el contenido de colágeno puro (yield $\mathrm{mg}$ ), el ratio $\mathrm{C}: \mathrm{N}$, el \% $\mathrm{y}$ el $\delta^{13} \mathrm{C}$. La concentración relativa de colágeno (expresado en \% del peso original del hueso o \% yield) en una muestra moderna es ca. 22\% (Jacobi et al. 2006). El ritmo de disminución de \% yield durante el enterramiento de la muestra depende de su edad y de las condiciones climáticas tras su deposición (van Klinken 1999). Según Ambrose (1990: 447), las muestras con menos de $3,5 \%$ de colágeno suelen tener valores anómalos en otros parámetros isotópicos y elementales (e.g. $\% \mathrm{C}, \mathrm{C}: \mathrm{N}$ ratios, $\delta^{13} \mathrm{C}$ ) que revelan un estado de conservación del colágeno defectivo y/o limitado (contaminación, alteraciones diagenéticas). En el Oxford Radiocarbon Accelerator Unit (ORAU) el límite es 1\% (van Klinken 1999).

El contenido de colágeno puro de la muestra (yield $\mathrm{mg}$ ) es una medida absoluta (expresada en mg) de la concentración de colágeno. Cuanto menor es la cantidad de colágeno puro gelatinizado extraído de una muestra, mayores son los efectos de la contaminación. En el ORAU el límite establecido es $10 \mathrm{mg}$ (Bronk-Ramsey et al. 2004a). La concentración relativa de carbono en el colágeno gelatinizado es el \%C. Según van Klinken (1999) cuando $\% \mathrm{C}=\sim 35 \pm 9$ las muestras están bien conservadas. Por encima de este rango hay incorporación de carbono orgánico (contaminación) y, por debajo, sustancias inorgánicas en la gelatina (alteración diagenética).

Según DeNiro y Weiner (1988: 2198; también Ambrose 1990) las muestras óseas con una relación carbono-nitrógeno ( $\mathrm{C}: \mathrm{N}$ ratio) comprendida entre 2,9 y 3,6 presentan un buen estado de conservación. Para Schoeninger et al. (1989) las muestras idóneas tienen un $\mathrm{C}: \mathrm{N}$ ratio entre 2,6 y 3,4. En el ORAU el rango de valores establecido es 3,1 y 3,5 (van Klinken 1999). A pesar de estas diferencias, todos los autores señalan que un valor superior a 4 indica alteraciones diagenéticas, o una proporción alta de carbono exógeno (por ejemplo, ácidos húmicos), que comprometen la fiabilidad de los resultados obtenidos (Hedges y van Klinken 1992).
El ratio delta del carbono $13, \delta^{13} \mathrm{C}$ del colágeno, varía en función de factores medioambientales: la concentración de $\mathrm{CO}^{2}$ en la atmósfera, la posición en la cadena trófica de la especie datada (carnívoro, herbívoro...), su régimen alimenticio (es importante conocer el patrón fotosintético de las plantas que constituyen la dieta de los herbívoros), y el tipo de hábitat (bosque, estepa...). La identificación de la especie es, pues, un requisito imprescindible para interpretar los resultados obtenidos. Según van Klinken (1999) en climas templados, no marinos, y no $\mathrm{C}_{4}$, los valores $\delta^{13} \mathrm{C}$ varían entre $-19 \%$ y $-22 \%$. Un valor inferior a este rango (e.g. -24\%o) puede indicar que la muestra está contaminada con ácidos húmicos, procedentes de la descomposición de las plantas, sobre todo si presenta unos valores altos de $\mathrm{C}: \mathrm{N}$.

Estos parámetros químicos permiten, en teoría, identificar las muestras arqueológicas (óseas) contaminadas con carbono exógeno (moderno o fósil). Sin embargo, en la práctica algunas dataciones incongruentes con la secuencia estratigráfica y con la adscripción cultural del nivel datado, ¿contaminadas?, tienen valores isotópicos y elementales coherentes con los rangos descritos más arriba. En consecuencia estos parámetros químicos no son capaces de detectar todas las muestras contaminadas, aunque son útiles a la hora de identificar las más anómalas, i.e. las muestras óseas con un $\mathrm{C}: \mathrm{N}$ ratio muy alto, una concentración relativa de carbono, $\% \mathrm{C}$, superior a $\sim 44 \%$ y un valor muy negativo de $\delta^{13} \mathrm{C}$. Estos parámetros no permiten determinar con absoluta seguridad si una muestra está contaminada o no, ni cuantificar el grado de contaminación de esa muestra (van Klinken 1999: 692). En cambio, su evaluación permite, sin lugar a dudas, identificar y descartar algunas muestras potencialmente contaminadas. Sin embargo, estos parámetros no suelen incluirse en la publicación de las dataciones, lo que imposibilita su evaluación, several thousand radiocarbon determinations are available on various databases. However, deciphering quite which of them are reliable and which not, is an often impossible task, because the key information we require in order to diagnose this, such as a pre-treatment method description and basic analytical data, is almost always unavailable (Higham et al. 2009: 1257-1258). 


\section{LA PERVIVENCIA NEANDERTAL/ MUSTERIENSE EN EL SUR DE LA PENÍNSULA IBÉRICA: LOS CASOS DE GORHAM'S CAVE Y LA SIMA DE LAS PALOMAS DEL CABEZO GORDO}

La cueva de Gorham (Gibraltar, Reino Unido) es, sin lugar a dudas, el yacimiento "transicional" más datado de la PI y el principal baluarte de una pervivencia Musteriense-neandertal en el sur de este territorio. Ha sido objeto de tres excavaciones arqueológicas, unas dirigidas por J. d'A Waechter (1948-1954), en la entrada, y otras por el Natural History Museum (1995-1998) y el Gibraltar Museum (1997-continúan). Estas últimas intervenciones se han centrado en el porche-entrada y en el interior ( 40 $\mathrm{m}$ de la entrada).

Waechter dividió la secuencia estratigráfica ( $8 \mathrm{~m}$ de potencia) en 26 niveles arqueológicos adscritos al Paleolítico medio-Musteriense (G-H, $\mathrm{K}-\mathrm{U})$ y a un Paleolítico superior "indeterminado" (B, D-F) (Barton 1988). Se recuperaron unas 10.000 piezas líticas, $c a$. $92 \%$ de los niveles musterienses. En la actualidad sólo se conservan 250 piezas de la colección original (Barton 1988). Los niveles G (5.233 piezas líticas, hoy 54) y D (568 artefactos, hoy 5) fueron datados respectivamente en \pm 48.500 BP y \pm 28.000 BP.

En el porche de la cueva la secuencia estratigráfica es más compleja. Barton et al. (1999: 16; Pettitt y Bailey 2000) han correlacionado los niveles $\mathrm{B}, \mathrm{D}$ y $\mathrm{E}$ de Waechter con las unidades (denominadas context) 8, 9 y 11 de las excavaciones modernas. Las fechas C14 de la unidad 9 (nivel D), entre 29.000 y 30.000 años BP, no difieren estadísticamente de las obtenidas en el nivel D. El escaso material arqueológico recuperado se considera compatible con una atribución del nivel al Paleolítico superior (Barton 2000: 217-218; Pettitt y Bailey 2000: 156). A su vez, los autores han correlacionado la unidad 22, inscrita sin mayores problemas en un Musteriense sensu lato, con el nivel G de Waechter. Las dataciones C14 entre 45.000 y 52.000 años BP (Tab. 2) coinciden grosso modo con las del nivel $\mathrm{G}$.

Por encima de la unidad 22 se han identificado dos unidades arqueológicas adscritas al Paleolítico medio-Musteriense (de techo a muro context 18 y 19). La unidad 18 y la transición 18/19 han sido datadas por radiocarbono (Tab. 2): $42.200 \pm$
$1.100(\mathrm{OxA}-7791), 23.800 \pm 600(\mathrm{OxA}-7979) \mathrm{y}$ $42.800 \pm 2.100($ OxA-8542). A simple vista se observa una discrepancia entre OxA-7979 y el resto. Según Pettitt y Bailey (2000: 158) OxA7979 is clearly too young and must therefore be intrusive from above... This is not surprising given that all of these samples are small, isolated lumps of charcoal which are prone to stratigraphic mobility. Aún aceptando la hipótesis de los desplazamientos (OxA-7979), surge la cuestión de la procedencia de la muestra. Ningún nivel superior tiene una fecha de edad comparable: los niveles arqueológicos adscritos al Paleolítico superior están datados entre 29.000 y 30.000 años $\mathrm{BP}$, mientras que la indeterminada unidad 24 tiene una edad de 32.280 años (OxA-7857). Una interpretación alternativa es la contaminación de la muestra OxA-7979 con carbono moderno. Si se toma como edad mínima verdadera la media de OxA-7791 y OxA-8542 (i.e. 42.500 años BP), se puede calcular el porcentaje de contaminación necesario para explicar una desviación cronológica de 18.700 años (Tab. 1), en este caso el porcentaje de carbono moderno es igual a $\sim 5 \%$. Si se acepta la hipótesis de los desplazamientos el porcentaje de contaminación varía entre un $\sim 1,5 \%$ y un $\sim 2 \%$, dependiendo del nivel que consideremos como el originario, i.e. context 24 o 9 respectivamente.

La unidad 19 también ha sido datada por radiocarbono (Tab. 2): $31.900 \pm 1.400$ (OxA-8541), $43.800 \pm 1.300$ (OxA-8525), $47.900 \pm 2.100$ $(\mathrm{OxA}-205)$ y $46.700 \pm 1.900(\mathrm{OxA}-8526)$, las dos últimas obtenidas a partir de la misma muestra. De nuevo, se advierte una discordancia entre OxA-8541 y el resto. Según Pettitt y Bailey (2000: 159), the underestimation of OxA-8541 demonstrates again the problems of intrusiveness in cave deposits. De forma paralela al caso anterior, esta discrepancia puede interpretarse en términos de contaminación. De ser así, el porcentaje de contaminación necesario para que una datación de $c a$. 46.133 años (i.e. media de OxA-8525, OxA-205 y OxA-8526) experimente un rejuvenecimiento de \pm 14.000 años es inferior a un $2 \%$.

En resumen, las excavaciones modernas en el porche de Gorham's Cave corroboran, en general, la secuencia crono-estratigráfica y cultural propuesta por Waechter. Las últimas ocupaciones musterienses bien documentadas en este sector desde un punto de vista tecno-tipológico y cronológico (Pettitt y Bailey 2000) se corresponden con 


\begin{tabular}{|c|c|c|c|c|}
\hline Sector & Nivel & Ref. Lab & Edad BP & $\delta \mathbf{C}^{13}$ \\
\hline \multirow{12}{*}{ ENTRADA } & Cont 18 & OxA-7791 & $42.200 \pm 1100$ & $-23,9$ \\
\hline & Cont 18 & OxA-7979 & $23.800 \pm 600$ & $-21,7$ \\
\hline & Cont 18/19 & OxA-8542 & $42.800 \pm 2100$ & $-24,4$ \\
\hline & Cont 19 & OxA-8541 & $31.900 \pm 1400$ & $-24,9$ \\
\hline & Cont 19 & OxA-8525 & $43.800 \pm 1300$ & $-20,9$ \\
\hline & Cont 19 & OxA-205 & $47.900 \pm 2100$ & $-23,4$ \\
\hline & Cont 19 & OxA-8526 & $46.700 \pm 1900$ & $-23,7$ \\
\hline & Cont 22 & OxA-6075 & $45.300 \pm 1700$ & $-25,2$ \\
\hline & Cont $22 d$ & OxA-7790 & $51.700 \pm 3300$ & $-24,5$ \\
\hline & $\mathrm{G}$ & GrN 1556 & $49.200 \pm 3200$ & - \\
\hline & $\mathrm{G}$ & GrN 1473 & $47.700 \pm 1500$ & - \\
\hline & $\mathrm{G}$ & GrN 1678 & $>47.000$ & - \\
\hline \multirow{22}{*}{ INTERIOR } & IV & Beta-196785 & $26.070 \pm 360$ & $-25,6$ \\
\hline & IV & Beta-196784 & $28.360 \pm 480$ & $-26,1$ \\
\hline & IV & Beta-185344 & $27.020 \pm 480$ & $-25,0$ \\
\hline & IV & Beta-196786 & $29.910 \pm 600$ & $-24,7$ \\
\hline & IV & Beta-196787 & $31.480 \pm 740$ & $-23,7$ \\
\hline & IV & Beta-196792 & $30.310 \pm 620$ & $-24,7$ \\
\hline & IV & Beta-185345 & $23.780 \pm 540$ & $-25,0$ \\
\hline & IV & Beta-196775 & $24.010 \pm 320$ & $-24,0$ \\
\hline & IV & Beta-184045 & $31.110 \pm 460$ & $-23,7$ \\
\hline & IV & Beta-196778 & $29.720 \pm 560$ & $-24,8$ \\
\hline & IV & Beta-196782 & $23.360 \pm 320$ & $-22,4$ \\
\hline & IV & Beta-196768 & $31.290 \pm 680$ & $-25,4$ \\
\hline & IV & Beta-196772 & $31.780 \pm 720$ & $-23,1$ \\
\hline & IV & Beta-196789 & $32.100 \pm 800$ & $-24,5$ \\
\hline & IV & Beta-196769 & $31.850 \pm 760$ & $-23,5$ \\
\hline & IV & Beta-196770 & $28.170 \pm 480$ & $-25,9$ \\
\hline & IV & Beta-184048 & $29.210 \pm 380$ & $-25,2$ \\
\hline & IV & Beta-196771 & $32.560 \pm 780$ & $-25,1$ \\
\hline & IV & Beta-196773 & $26.400 \pm 440$ & $-23,2$ \\
\hline & IV & Beta-196791 & $28.570 \pm 480$ & $-25,2$ \\
\hline & IV & Beta-196779 & $29.400 \pm 540$ & $-25,4$ \\
\hline & IV & Beta-196776 & $30.560 \pm 720$ & $-24,5$ \\
\hline
\end{tabular}

Tab. 2. Dataciones de radiocarbono de Gorham's Cave (Gibraltar, Reino Unido) según Pettitt y Bayley (2000) y Finlayson et al. (2006). $\delta^{13} \mathrm{C}$ : ratio delta del carbono 13. En negrita se muestran los valores anómalos según los rangos propuestos en la bibliografía (Ambrose 1990; van Klinken 1999; Maroto et al. 2012). Todos los niveles mencionados en la tabla pertenecen al Musteriense y las muestras son de carbón.

Trab. Prehist., 70, N. ${ }^{\circ}$ 2, julio-diciembre 2013, pp. 241-263, ISSN: 0082-5638

doi: $10.3989 /$ tp.2013.12111 
las unidades 18 , datada en \pm 42.000 años BP, y 19 en \pm 46.000 años BP. Por encima de estos niveles se han identificado varias ocupaciones adscritas a un Paleolítico superior indeterminado, datadas entre 29.000 y 30.000 años BP.

La secuencia estratigráfica definida en el interior de la cueva ( $\sim 40 \mathrm{~m}$ de la entrada) contradice en muchos aspectos los resultados obtenidos en la entrada. Hasta el momento, se han identificado 4 niveles arqueológicos adscritos al Paleolítico medio y superior. Según Finlayson et al. (2006), la unidad III (240 piezas líticas) está formada por 3 niveles de ocupación (dos solutrenses y uno magdaleniense) datados entre $10.880 \pm 80 \mathrm{BP}$ (Beta-185343) y $18.440 \pm 160$ BP (Beta-104042). Bajo este nivel, se ha identificado el nivel IV Musteriense (222 artefactos líticos) (Giles Pacheco et al. 2012) con 22 dataciones de radiocarbono entre $23.360 \pm 320$ BP (Beta-196782) y $32.560 \pm 780$ BP (Beta-196771, Tab. 2). Según Finlayson et al. (2006: 850-851), esto permite afirmar that Neanderthals occupied the site until $28 \mathrm{kyr} B P$ and possibly as recently as $24 \mathrm{kyr} B P$. The evidence in support of the $24 \mathrm{kyr} B P$ date is more limited than for $28 \mathrm{kyr} B P$, which is taken as the latest wellsupported occupation date... the dates suggest a favoured location that was visited repeatedly over many thousands of years.

Esta interpretación, incoherente con los resultados obtenidos en el porche de la cueva, ha sido objeto de duras críticas (Zilhão y Pettitt 2006). Sin embargo, hasta la fecha no se ha solventado la contradicción que representa una ocupación musteriense-neandertal en el fondo de la cueva, contemporánea (en términos absolutos/numéri$\cos$ ) de un asentamiento del Paleolítico superior indeterminado- ¿sapiens? en el porche. En general, aceptamos las críticas de Zilhão y Pettitt (2006) y consideramos que la interpretación de Finlayson et al. $(2006,2008)$ no respeta los principios estratigráficos descritos más arriba.

La atribución cultural del nivel datado se basa en 222 artefactos líticos (Giles Pacheco et al. 2012): 43 bases naturales (i.e. manuports y percutores), 12 núcleos (dos clasificables como choppers), 154 productos de lascado (lascas sensu lato) y 12 útiles (denticulados, muescas y raederas, sin tipologías del PS). Según Giles Pacheco et al. (2012: 161), it may be concluded from both the Technical Operative Themes... and from the final configuration of the tools... that the site illustra- tes an industrial complex that can be classified within the Mousterian techno-complex. Se trata, en cualquier caso, de un conjunto lítico "paupérrimo" (Barandiarán et al. 1996) con un potencial diagnóstico limitado.

La asociación de las muestras datadas y del evento que se quiere datar es dudosa. El nivel IV tiene una potencia media de $1 \mathrm{~m}$ y una extensión máxima de $20 \mathrm{~m}^{2}$ (Finlayson et al. 2006: 851), lo que supone una densidad media de $\sim 11$ artefactos líticos por $\mathrm{m}^{3}$. Sin embargo, hasta el momento no se ha publicado ninguna prueba convincente (e.g. distribución espacial de las piezas líticas, remontajes líticos...) que corrobore la asociación espacial de las muestras datadas con la industria lítica y aclare si estamos ante uno o varios niveles de ocupación y ante artefactos en posición primaria o secundaria. Además hay bastantes ejemplos en la bibliografía de transporte de materiales por solifluxiones y removilizaciones en el interior de las cavidades.

La distribución espacial de las muestras datadas revela varias "inversiones cronológicas" (en un paquete de $\sim 50 \mathrm{~cm}$ de potencia), inconsistentes con la hipótesis de una perduración musterienseneandertal hasta $c a$. 28/24.000 años BP, permitiendo, a su vez, una interpretación del registro en términos de contaminación. Ninguna datación ca. 24.000 años BP se localiza a techo del nivel. La muestra 23 (Beta-196782, $23.360 \pm 320$ ) está rodeada por 10 dataciones $>30.000$ años BP, lo que implica un porcentaje de contaminación de $\sim 3 \%$. Ahora bien, si se toma como edad mínima verdadera la edad media del último nivel musteriense documentado en el porche de la cueva (context 18, \pm 42.500 años BP), el porcentaje de contaminación necesario para explicar el rango de dataciones obtenido en el nivel IV (i.e. $\sim 23.360-32.100$ años BP) varía entre un $\sim 1,5 \%$ y un $\sim 5,3 \%$, aceptando la hipótesis no demostrada de que todas la fechas obtenidas en este nivel datan la ocupación musteriense. Estaríamos ante un caso insólito donde todas las fechas de radiocarbono estarían contaminadas con carbono moderno. No obstante los porcentajes de contaminación necesarios para explicar estas desviaciones son asumibles en Gorham's Cave (por ejemplo OxA-7979 y OxA-8541) y en otros yacimientos de la PI datados en la transición (i.e. al límite de aplicabilidad del radiocarbono).

La comparación de las estimaciones C14 obtenidas en los laboratorios Beta Analytic y ORAU 
para el Musteriense de la Península Ibérica revela importantes discrepancias entre ambos laboratorios que difícilmente pueden explicarse a partir del contexto arqueológico. Como se observa en la figura 4 el $\sim 68 \%$ de las dataciones $\mathrm{C} 14$ realizadas en Beta Analytic son inferiores a 35.000 años BP, solo el $\sim 13 \%$ de las fechas son superiores a 40.000 años BP y apenas una supera la barrera del 45.000. En cambio, en el ORAU el 62,5\% de las dataciones $\mathrm{C} 14$ son superiores a 40.000 años BP, y el 37,5\% superan la barrera del 45.000 . Esto podría explicar las diferencias advertidas en Gorham's Cave entre la unidad IV (Beta Analytic, entre 24.000 y 32.000 años BP) y el sector entrada (ORAU, >42.000 años BP). Estas variaciones son estadísticamente significativas $\left(\mathrm{X}^{2}: 40,535 \mathrm{p}\right.$ valor $<0,000, \mathrm{~V}$ de Cramer: 0,572) y pueden estar relacionadas con los métodos de pretratamiento utilizados en cada caso (Longin, Ultrafiltración/ $\mathrm{ABA}, \mathrm{ABOx}) \mathrm{y} / \mathrm{o}$ con el instrumental background específico de cada laboratorio. Las diferencias cronológicas entre estos laboratorios han sido constatadas en los yacimientos asturianos de El Sidrón o La Güelga. En la cueva de El Sidrón las existentes entre Beta Analytic-Longin por una parte, y ORAU-Ultrafiltración y Gif-sur-YvetteNinhydrina por otra, se pueden estimar en $c a$. 10.000-15.000 años (lo que implica unos niveles de contaminación entre $\sim 0,5 \%$ y $\sim 1,5 \%$ ) (Torres et al. 2010; Santamaría 2012; Wood et al. 2013). Si se tiene en cuenta que la acumulación de los restos óseos (neandertales) y líticos (Musteriense de denticulados) en la Galería del Osario fue el resultado de un evento natural y cultural único (Santamaría et al. 2010; Santamaría et al. 2011), las implicaciones culturales de este yacimiento varían notablemente si lo situamos en $\sim 35 / 40.000$ años BP (estimaciones C14 de Beta Analytic; i.e. en plena transición PM-PS) o en 48-50.000 años $\mathrm{BP}$ (estimaciones $\mathrm{C} 14$ de ORAU y Gif sur Yvette; i.e. antes de la llegada de $H$. sapiens a la PI).

En resumen, el nivel IV de Gorham's Cave carece de garantías crono-estratigráficas y tecnotipológicas (distribución espacial de los artefactos líticos) suficientes para sustentar la hipótesis de una pervivencia musteriense en el sur de la PI hasta 2428.000 años BP. A día de hoy, las únicas evidencias musterienses bien documentadas en la cueva están datadas en $>42.000$ años BP, y se corresponden con el nivel $\mathrm{G}$ de Waechter y con las unidades 18-19 y 22 de las excavaciones modernas (sector entrada).
El sector que nos interesa aquí de la Sima de las Palomas del Cabezo Gordo (Torre Pacheco, Murcia) se localiza en la parte superior de una sima brechoide de $18 \mathrm{~m}$ de profundidad, parcialmente vaciada durante el siglo XIX (minería) y excavada desde 1992 por M. J. Walker y J. Gibert. Se llevan recuperados más de 100 restos óseos humanos, pertenecientes a la especie $H$. neanderthalensis, y algunas piezas líticas adscritas a un Musteriense sensu lato.

Walker et al. (2008) publicaron las 6 primeras dataciones radiométricas de este sector (denominado corte superior): 2 huesos quemados (uno indeterminado y otro de conejo) procedentes de los niveles $2 \mathrm{f}$ y 21 , datados por radiocarbono (OxA10666: $34.450 \pm 600$ BP y OxA-15423: 35.030 \pm 270 BP respectivamente, Tab. 3), 2 huesos de fauna indeterminados procedentes de los niveles $2 \mathrm{i}$ y 21 , datados por U/Th (APSLP4: $43.800 \pm 750$ y APSLP6: $51.000 \pm 1.250$ respectivamente), 1 metacarpo neandertal datado por U/Th cuya procedencia es incierta (vid infra, núm. de inventario Palomas 96, APSLP1: $54.000 \pm 3.850$ BP) y 1 muestra de sedimento, procedente del nivel $2 \mathrm{k}$, datada por OSL (X2509: $54.700 \pm 4.700$ BP). Según los autores, los resultados radiométricos obtenidos en este sector permiten concluir que the human remains from the Sima de la Palomas in southeastern Iberia therefore document the presence of Neandertals, relatively late in the Middle Paleolithic [i.e. 35.000 años BP]. They help to substantiate that the Middle Paleolithic of the region was the product of Neandertals, even though diagnostic human remains associated with the very latest phases of this technocomplex in Europe remain elusive. Esto unido a ciertas características anatómicas "modernas" que presentan los restos fósiles neandertales de Cabezo Gordo, les lleva a plantear que this pattern may be result of genetic drift in relative isolation, directional change or, perhaps more likely, population contact to the north [i.e. con $H$. sapiens-auriñaciense] (Walker et al. 2008: 20635). La interpretación cronológica, cultural y antropológica propuesta por Walker y otros se puede resumir como sigue: (fechas \pm 35.000 años $\mathrm{BP})+($ características anatómicas modernas de los restos fósiles neandertales) $=$ hibridación .

Los argumentos cronoestratigráficos que sustentan esta hipótesis son, a nuestro entender, poco consistentes con los datos publicados hasta la fecha. Hay imprecisiones-omisiones de orden

Trab. Prehist., 70, N. ${ }^{\circ}$ 2, julio-diciembre 2013, pp. 241-263, ISSN: 0082-5638

doi: $10.3989 /$ tp.2013.12111 


\begin{tabular}{|c|c|c|c|c|c|c|}
\hline Capa & $\begin{array}{c}\text { Restos } \\
\text { humanos }\end{array}$ & $\begin{array}{l}\text { Artefactos } \\
\text { líticos }\end{array}$ & Método & Referencia & Muestra & Edad BP \\
\hline $2 \mathrm{a}$ & 2 & - & & & & \\
\hline $2 b$ & 4 & 14 & & & & \\
\hline $2 \mathrm{c}$ & 39 & 2 & U/Th & APSLP1 & Hueso neandertal & $54.000 \pm 3850$ \\
\hline $2 d$ & 7 & 7 & $\mathbf{U} / \mathbf{T h}$ & APSLP1 & Hueso neandertal & $54.000 \pm 3850$ \\
\hline $2 \mathrm{~d}-2 \mathrm{e}$ & 11 & - & & & & \\
\hline $2 \mathrm{e}$ & 23 & - & U/Th & APSLP1 & Hueso neandertal & $54.000 \pm 3850$ \\
\hline $2 \mathrm{f}$ & 6 & 9 & AMS & OxA-10666 & Hueso quemado & $34.450 \pm 600$ \\
\hline $2 g$ & 4 & 8 & & & & \\
\hline $2 \mathrm{~h}$ & 43 & 11 & U/Th & APSLP1 & Hueso neandertal & $54.000 \pm 3850$ \\
\hline $2 \mathrm{i}$ & 3 & 25 & $\mathrm{U} / \mathrm{Th}$ & APSLP4 & Hueso & $43.800 \pm 750$ \\
\hline $2 \mathrm{j}$ & - & 6 & & & & \\
\hline $2 \mathrm{k}$ & 2 & - & OSL & $\mathrm{X} 2509$ & Sedimento & $54.700 \pm 4700$ \\
\hline 21 & - & - & $\begin{array}{l}\text { AMS } \\
\text { U/Th }\end{array}$ & $\begin{array}{c}\text { OxA-15423 } \\
\text { APSLP6 }\end{array}$ & $\begin{array}{c}\text { Hueso quemado } \\
\text { Hueso }\end{array}$ & $\begin{array}{l}35.030 \pm 270 \\
51.000 \pm 1250\end{array}$ \\
\hline $2 \mathrm{~m}$ & 1 & - & & & & \\
\hline $2 n$ & 1 & - & & & & \\
\hline 20 & 2 & - & & & & \\
\hline Capa & Referencia & Edad & Yield (mg) & $\%$ Yield & $\% \mathrm{C}$ & $d^{13} \mathrm{C}$ \\
\hline $2 f$ & OxA-10666 & $34.450 \pm 600$ & 39,25 & 5,3 & 8 & $-21,0 \%$ \\
\hline 21 & OxA-15423 & $35.030 \pm 270$ & 6,30 & 2,5 & 40,9 & $-22,3 \%$ \\
\hline
\end{tabular}

Tab. 3. Resumen estratigráfico, cronológico y arqueológico del Cabezo Gordo (Torre Pacheco, Murcia). Restos humanos: número de restos óseos neandertales (Walker et al. 2008, supporting information); Artefactos líticos: número de restos líticos por capa (Walker et al. 2006); Dataciones (Walker et al. 2008), en negrita se localiza APSLP1 (Walker et al. 2008 y supporting information). La parte inferior de la tabla reproduce los parámetros químicos de las dataciones de radiocarbono (Walker et al. 2008 supporting information). En negrita se muestran los valores anómalos según los rangos publicados (Ambrose 1990; van Klinken 1999; Maroto et al. 2012). Todas las capas citadas en la tabla pertenecen al Musteriense. U/Th $=$ Uranio/Torio, AMS = Radiocarbono Accelerator Mass Spectrometry, OSL = Optically Stimulated Luminescence.

estratigráfico. Walker et al. (2008) dividen la unidad litoestratigráfica 2 en otras que denominan niveles (e interpretan como tales). No obstante previamente (Walker y Gibert 2002a, 2002b, 2004, 2005; Walker et al. 2006) expusieron que esta denominación ( $2 \mathrm{a}, 2 \mathrm{~b}, 2 \mathrm{c} \ldots$. hasta la capa $2 \mathrm{o}$ ) tenía un significado metodológico/procedimental -la división artificial de la unidad litoestratigráfica 2 en capas sucesivas de $5 \mathrm{~cm}$ de espesor (Gibert y Walker 1999: 13-14 y 16)- y no estratigráfico/sedimentológico (Walker et al. 2008). Según Walker et al. (2008: 20631), la pervivencia musterienseneandertal en Cabezo Gordo se documenta en los niveles (capas) superiores (por encima de 2 $\mathrm{m})$, posteriores desde un punto de vista cronoes- tratigráfico a la entrada de una masa caótica de bloques en forma de cono (Walker et al. 2008: 20631) que rellena la mitad occidental del sector, por encima del "nivel" $2 \mathrm{~m}$. El cono de derrubios permite dividir la secuencia estratigráfica de este sector en dos grandes unidades: previa y posterior al colapso. Lo que plantea dudas son los argumentos estratigráficos que justifican la división del conjunto superior (i.e. capas 2a-21, posteriores al derrumbe y datadas entre \pm 35.000 años BP y \pm 54.000 años BP) en diferentes unidades estratigráficas-niveles (niveles 2a-21). Si las capas 2a-2l forman parte de la misma unidad litoestratigráfica, la interpretación cronológica de la unidad debería hacerse de forma conjunta. 
Las incertidumbres estratigráficas son dos. Según Walker et al. (2008: 20632) la muestra APSLP1 (metacarpo neandertal, núm. inventario Palomas 96) procede de la capa 2e (i.e. a techo de OxA-10666, Tab. 3), pero en la supporting information se asigna al $2 \mathrm{~h}$ (i.e. a muro de OxA-10666). Sorprendentemente los 9 metacarpos neandertales inventariados como Palomas 96 (Walker et al. 2008 supporting information) se asignan a los niveles $2 \mathrm{~d}$ (5 ejemplares) y $2 \mathrm{c}$ (4 ejemplares), i.e. ambos por encima de OxA10666. En resumen, la misma publicación localiza la muestra APSLP1 en cuatro niveles-capas diferentes $(2 \mathrm{c}, 2 \mathrm{~d}, 2 \mathrm{e}$ y $2 \mathrm{~h})$. La posición estratigráfica de esta muestra es crucial, ya que se trata del único resto humano datado directamente $(54.000$ $\pm 3.850)$. La segunda incertidumbre surge ante la proyección estratigráfica de las muestras APSLP6 y X2509 ( 30 cm de distancia en planta), que no se corresponde con la posición relativa de los niveles-capas asignados a cada una (Walker et al. 2008): X2509 (capa 2k) se localiza a una cota inferior (i.e. a muro) de APSLP6 (capa 21), cuando según la sucesión lógica de capas-niveles X2509 (capa 2k) debería situarse a techo de APSLP6 (capa 21).

La interpretación cronológica propuesta por Walker et al. (2008) se fundamenta, a nuestro juicio, en una evaluación interesada de las fechas radiométricas, cuyo objetivo es sustentar la hipótesis de la hibridación neandertal-sapiens. Esto implica considerar a las fechas U/Th y OSL subsidiarias de las del radiocarbono, lo que no deja de ser paradójico en un yacimiento cuyo único resto óseo neandertal datado directamente (APSLP1, $54.000 \pm 3.850$ ) lo fue mediante U/ Th. Walker et al. (2008: 20632) afirman, primero, que these U-series [refiriéndose a APSLP1 y APSLP6] estimates that the samples were closed systems, which cannot be verified. They should therefore be regarded only as corroborating the $C 14 \mathrm{y}$, en el párrafo siguiente, que the sample [refiriéndose a la datación OSL X2509] provided an age estimate of 54,700 $\pm 4,700 \mathrm{cal} \mathrm{BP}$ for these sediments. This determination increases confidence in the U-series dates for stratigraphically similar specimens and hence in the age of the stratigraphically younger deposits dated by $C 14$. Se puede aceptar que la fecha OSL corrobore las dataciones U/Th, pero no que todas ellas confirmen a su vez las fechas de radiocar- bono. Tres dataciones superiores a 50.000 años BP (APSLP1, APSL6 y X2509 con unas desviaciones típicas entre 1.250 y 4.700 años) no pueden corroborar dos fechas de \pm 35.000 años BP, máxime cuando estas presentan unos valores anómalos en $\% \mathrm{C}$ y $\mathrm{C}: \mathrm{N}$ ratio (Tab. 3 sección inferior) que cuestionan su fiabilidad. Además, aunque los autores no ubican estratigráficamente la muestra OxA-15423, su asignación al nivelcapa 21 la sitúa a la misma cota que APSLP6 (i.e. a muro de la secuencia estratigráfica, y por debajo del resto de dataciones, Tab. 3). Si se toma como edad mínima verdadera del nivel la media de las dataciones U/Th y OSL (i.e. 50.875 años $\mathrm{BP})$, el porcentaje de contaminación necesario para explicar las fechas de radiocarbono (una vez calibradas según Calpal 07 e Intcal09, Weninger y Jöris 2008; Reimer et al. 2009) es igual a $\sim 0,5 \%$. En este contexto, la explicación más razonable (desde un punto de vista cronoestratigráfico) es, a nuestro entender, la contaminación de las muestras óseas con carbono moderno. Esto explicaría por otra parte los valores anómalos del ratio C:N. Así según los datos publicados hasta la fecha, y a pesar de las graves incoherenciascontradicciones estratigráficas que cuestionan su fiabilidad, la edad de los restos óseos neandertales de Cabezo Gordo no puede ser posterior a 50.000 años BP.

\section{SOBRE EL MUSTERIENSE TARDÍO DE LA REGIÓN CANTÁBRICA}

En los últimos años, la región cantábrica ha sido incluida en la lista de posibles refugios neandertales/musterienses (Baena et al. 2004; Baena et al. 2005; Baena et al. 2006; Baena y Carrión 2006; Baena et al. 2012), en ocasiones, como en la cueva de El Esquilleu (Cantabria), hasta $c a$. 23.000 años BP (Baena et al. 2012). Esta hipótesis se asienta una vez más en las dataciones absolutas/numéricas obtenidas en los yacimientos musterienses asturianos de la La Güelga, El Conde y El Sidrón y sobre todo en el citado de El Esquilleu (Baena et al. 2012: 200). La Güelga, datado inicialmente en ca. 32.000 años BP (Menéndez et al. 2005), y El Sidrón (cuyas estimaciones C14 oscilan entre 10.000 y 49.000 años BP, Torres et al. 2010; Torres et al. 2011) han vuelto a datarse

Trab. Prehist., 70, N. ${ }^{\circ}$ 2, julio-diciembre 2013, pp. 241-263, ISSN: 0082-5638

doi: $10.3989 /$ tp.2013.12111 
recientemente en más de 45/50.000 años BP (Menéndez et al. 2009, Quesada y Menéndez 2009; Wood et al. 2013), por lo que deben ser excluidos de la lista de yacimientos musterienses tardíos (i.e. posteriores a 35.000 años BP).

Los datos publicados hasta la fecha de la cueva de El Conde (Arbizu et al. 2005a, 2009; Arbizu et al. 2005b; García et al. 2006; Adán y Arsuaga 2007; López-García et al. 2011) no permiten formular grandes teorías sobre la evolución cronocultural de la secuencia (Rasilla y Santamaría, 2013). El Musteriense ha sido identificado en las tres zonas excavadas: Galería A, nivel N2a1, datado en $24.680 \pm 160$ (Beta 210117) y $39.960 \pm$ 470 (Beta 210571, Arbizu et al. 2005a, 2009); Plataforma, nivel N20a, datado en $37.710 \pm 470$ (Beta 230416) y $38.250 \pm 390$ (Beta 210572) (Arbizu et al. 2009; López-García et al. 2011); Trinchera, nivel N103, datado en $28.280 \pm 230$ BP (Beta 223305, considerada por Arbizu et al. 2009 como rejuvenecida) y nivel N104, datado en $39.110 \pm 520 \mathrm{BP}$ (Beta 237328) (Arbizu et al. 2009; López-García et al. 2011). Las estimaciones C14 Beta 210117 y Beta 223305 deben ser consideradas anómalas por ser muestras intrusivas o contaminadas con carbono moderno. Ello sitúa el Musteriense final de El Conde entre 38.000 y 40.000 años BP. Teniendo en cuenta que en la PI las estimaciones $\mathrm{C} 14$ realizadas por Beta Analytic no suelen superar la franja del 40.000 BP (Fig. 4), es muy posible que estos niveles sean en realidad más antiguos.

La amplia secuencia musteriense de la cueva del Esquilleu (Cillorigo de Liébana, Cantabria) está datada entre $>60.000$ y ca. 19.000 años BP (Maroto et al. 2012, Tab. 4). Según Baena et al. (2012: 202) el Esquilleu has a sequence that has classical Mousterian dates in the lower levels and continues into "Würm III", with an unequivocal recent sequence between levels VI and IV (Level VI dates to $34.38 \mathrm{ka} \pm 670$; Level IV dates to $22.84 \mathrm{ka} \pm 280 / 250)$. The datings obtained for Esquilleu, although they could provide some shades in the overall coherence of the sequence, maintain, even strengthen, the uniqueness of the site in terms of continuation. A nuestro juicio, son esas sombras las que cuestionan, o al menos relativizan, el modelo cronológico propuesto por Baena et al. (2005; Baena et al. 2012). La secuencia estratigráfica del Esquilleu se divide de muro a techo en cuatro unidades litoestratigráficas: ESQ-

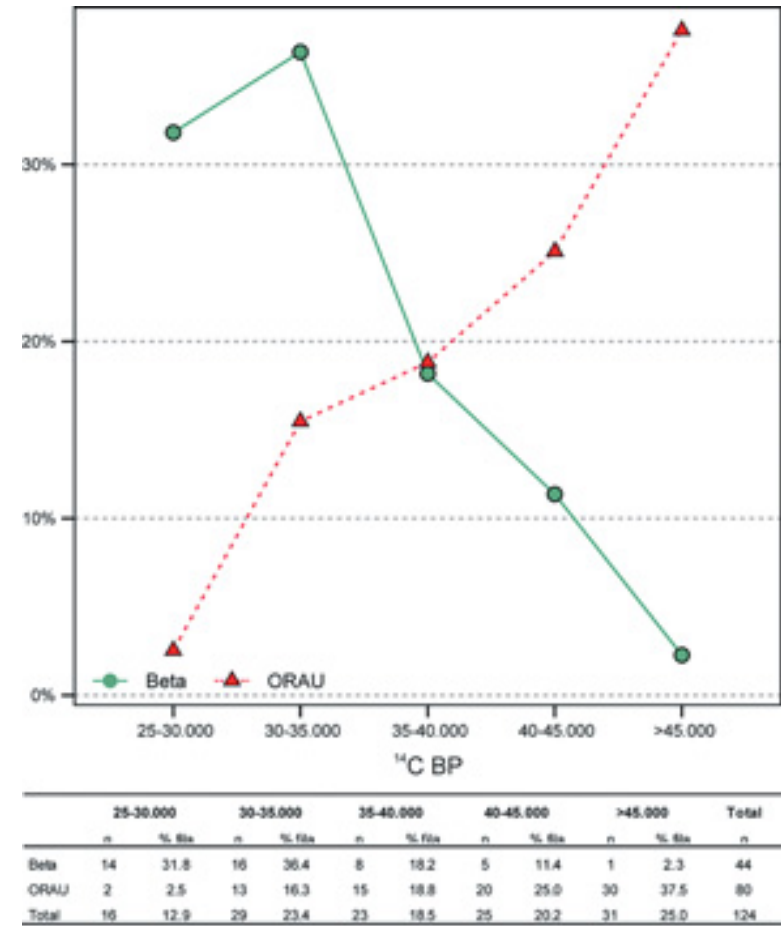

Fig. 4. Comparación de las estimaciones ${ }^{14} \mathrm{C}$ obtenidas en los laboratorios Beta Analytic (Beta) y Oxford Radiocarbon Accelerator Unit (ORAU) para el Musteriense de la Península Ibérica. En conjunto representan ca. 65\% de las dataciones ${ }^{14} \mathrm{C}$. Se excluyen todas las dataciones inferiores a 25.000 años BP.

D (niveles XXXI a XLI), ESQ-C (niveles XII a XXX), ESQ-B (niveles I a XI) y ESQ-A (Brecha y espeleotema culminante). Las unidades ESQ-C y ESQ-B son las que nos interesan. ESQ-C presenta "una sedimentación de abrigo rocoso con predominio de procesos de arroyada difusa". ESQ-B está "caracterizada por niveles ricos en clastos autóctonos con escasa matriz arenoso lutítica..., tiene su origen en una sedimentación de abrigo producida por procesos crioclásticos con aportes de arroyada difusa y flujos densos a techo" (Jordá et al. 2008: 36-37).

Las fechas obtenidas en el tramo inferior, ESQ$\mathrm{C}$, son bastante coherentes entre sí, habida cuenta del rango cronológico en el que nos movemos, con algunas salvedades. Disponemos de 4 dataciones para el nivel XIX, una superior a 54.600 años BP y tres en torno a 39.500 años BP. Todas se obtuvieron a partir de un mismo espécimen (tres pretratamientos diferentes ABA, PO y ABOX-SC, Tab. 4). Según Maroto et al. (2012: 20) las diferencias observadas 


\begin{tabular}{|c|c|c|c|c|c|c|c|c|c|}
\hline UL & Nivel & Muestra & Ref. Lab. & Edad BP & $\mathbf{P}$ & $\% \mathrm{C}$ & $\delta^{13} \mathbf{C}$ & $\mathrm{C}: \mathbf{N}$ & $\%$ Yield \\
\hline \multirow{13}{*}{$\mathrm{B}$} & III & Carbón & GrA-33829 & $3.640 \pm 90$ & A & 0,7 & $-26,3$ & & \\
\hline & III & Hueso & AA-29664 & $12.050 \pm 130$ & - & - & - & - & \\
\hline & III & Hueso & OxA-19967 & $19.300 \pm 100$ & UF & 46,9 & $-19,2$ & 3,4 & 5,1 \\
\hline & III & Hueso & OxA-19968 & $19.310 \pm 80$ & UF & 49,1 & $-19,4$ & 3,4 & 4,8 \\
\hline & IIIB & Hueso & OxA-19246 & $20.810 \pm 110$ & UF & 44,7 & $-19,4$ & 3,3 & 2,1 \\
\hline & IV & Carbón* & GrA-35064 & $22.840 \pm 280 / 250$ & $\mathrm{~A}$ & 15,2 & $-22,7$ & & \\
\hline & IV & Carbón* & GrA-35064 & $23.560 \pm 120$ & A & 15,2 & $-22,7$ & & \\
\hline & V & Carbón & GrA-35065 & $30.250 \pm 500 / 430$ & A & 49,7 & $-21,9$ & & \\
\hline & VIF & Carbón & AA37883 & $34.380 \pm 670$ & - & - & - & & \\
\hline & VI & Carbón & GrA-33816 & $40.110 \pm 500 / 420$ & $\mathrm{ABA}$ & 60,7 & $-25,2$ & & \\
\hline & VI & Hueso & OxA-19965 & $43.700 \pm 1400$ & UF & 46,2 & $-19,1$ & 2,4 & 1,3 \\
\hline & VI & Hueso & OxA-19966 & $44.100 \pm 1300$ & UF & 44,4 & $-19,3$ & 3,4 & 1,4 \\
\hline & XIF & Carbón & AA37882 & $36.500 \pm 830$ & - & - & - & & \\
\hline \multirow{14}{*}{$\mathrm{C}$} & XIII & Carbón & Beta149320 & $39.000 \pm 300$ & - & - & - & & \\
\hline & XVII & Carbón & OxA-X-2297-31 & $49.400 \pm 1300$ & ABOX-SC & 36,8 & $-23,2$ & & \\
\hline & XVII & Carbón & OxA-20320 & $52.600 \pm 1200$ & ABOX-SC & 74,7 & $-23,1$ & & \\
\hline & XVII & Carbón & OxA-20318 & $53.400 \pm 1300$ & ABOX-SC & 72,2 & $-24,5$ & & \\
\hline & XVII & Carbón & OxA-19993 & $>54.000$ & ABOX-SC & 66,2 & $-22,8$ & & \\
\hline & XVII & Carbón & OxA-20319 & $>58.500$ & ABOX-SC & 78,6 & $-21,7$ & & \\
\hline & XVIII & Carbón & OxA-11414 & $49.700 \pm 1600$ & - & - & - & & \\
\hline & XIX & Carbón* & OxA-19085 & $39.280 \pm 340$ & $\mathrm{ABA}$ & 60,0 & $-23,5$ & & \\
\hline & XIX & Carbón* & OxA-19086 & $>54.600$ & ABOX-SC & 62,9 & $-23,0$ & & \\
\hline & XIX & Carbón* & OxA-V-2284-29 & $39.600 \pm 400$ & $\mathrm{PO}$ & 52,3 & $-23,0$ & & \\
\hline & XIX & Carbón* & OxA-V-2284-30 & $39.650 \pm 450$ & $\mathrm{PO}$ & 49,4 & $-23,0$ & & \\
\hline & XXI-I & Carbón & OxA-20321 & $>59.600$ & ABOX-SC & 69,9 & $-21,7$ & & \\
\hline & XXId & Tierra quemada & Mad3299 (TL) & $51.034 \pm 5114$ & - & - & - & & \\
\hline & XXIb & Tierra quemada & $\operatorname{Mad} 3300(\mathrm{TL})$ & $53.491 \pm 5114$ & - & - & - & & \\
\hline
\end{tabular}

Tab. 4. El Musteriense de la cueva de El Esquilleu (Cillorigo de Liébana, Cantabria), dataciones absolutas (Baena et al. 2005; Baena et al. 2006; Baena et al. 2012, Jordá et al. 2008; Maroto et al. 2012). Unidad litoestratigráfica (UL) (Jordá et al. 2008). * Dataciones obtenidas sobre un mismo espécimen. Pretratamiento (P). A: sólo ácido (Maroto et al. 2012: 21, esta técnica solo se usa cuando la muestra es muy delicada para utilizar la secuencia completa ABA). ABA: ácido-base-ácido. ABOX-SC: ácido-base-ácido seguido de una fase de oxidación y combustión. PO: Plasma oxidación (técnica en fase experimental, Maroto et al. 2012: 21). UF: Ultrafiltración. \%C: Concentración relativa de carbono, $\delta^{13} C$ : ratio delta del carbono 13, C:N: ratio carbono-nitrógeno, \% yield: concentración relativa de colágeno. Van en negrita los valores anómalos según los rangos propuestos en la bibliografía (Ambrose 1990; van Klinken 1999; Maroto et al. 2012).

entre ABOX-SC (>54.600) y ABA-PO (ca. 39.500 años $\mathrm{BP})$ supports the conclusion that the ABOX-SC method removes contamination more effectively than others methods and yields therefore older results.
El porcentaje de contaminación necesario para explicar estas desviaciones es igual a $0,7 \%$. La fecha obtenida en el nivel XIII (Beta 149320, $39.000 \pm$ $300)$ debe ser rechazada por entrar en contradicción

Trab. Prehist., 70, N. ${ }^{\circ}$ 2, julio-diciembre 2013, pp. 241-263, ISSN: 0082-5638

doi: $10.3989 /$ tp.2013.12111 
con la secuencia crono-estratigráfica del yacimiento. Según las estimaciones C14 del ORAU la edad del suprayacente nivel VI es de $c a$. 44.000 años BP. Además, esta fecha procede de Beta Analytic, lo que cuestiona o relativiza su fiabilidad (Fig. 4). Según esto, la edad absoluta/numérica de ESQ-C (niveles XII a XXX) tiene que ser anterior a 45.000 años BP. Es muy probable incluso que la edad absoluta de estos niveles sea muy superior a la estimada por el radiocarbono ya que son muchas las fechas infinitas obtenidas.

Comentamos ahora las dataciones disponibles para el tramo superior, ESQ-B (niveles I a XI). La fecha obtenida en XIF (AA37882, $36.500 \pm$ 830) también debe ser rechazada por entrar en contradicción con la secuencia crono-estratigráfica del yacimiento. El nivel VI ha sido datado entre $34.380 \pm 670(\mathrm{AA} 37883)$ y $44.100 \pm 1300$ BP (OxA-19966). Según Baena et al. (2012) la edad de este nivel es 34.380 años BP. Sin embargo de las cuatro dataciones disponibles tres son superiores a 40.000 años y, además, el porcentaje de contaminación necesario para explicar las diferencias observadas en este nivel es tan solo del $\sim 1 \%$. Creemos que las estimaciones más fiables son, sin lugar a dudas, las obtenidas en el ORAU, que sitúan su edad en torno a 43/44.000 años BP. Los niveles V y IV han sido datados respectivamente en $c a .30 .000$ y ca. 23.000 años BP (Tab. 4). Las muestras solo han sido tratadas con ácido, porque eran muy delicadas para utilizar el tratamiento completo ABA (Maroto et al. 2012: 21), lo que cuestiona la fiabilidad de las dataciones. Además, las muestras del nivel IV presentan un \%C: 15,2 anómalo (Maroto et al. 2012: 21), muy inferior al rango (entre 50 y $70 \%$ ) aceptado en la bibliografía (Braadbaart et al. 2009), acentuando la incertidumbre sobre esas dataciones. Por último, el nivel III ha sido fechado entre $3.640 \pm 90$ y $20.810 \pm 110 \mathrm{BP}$. La datación GrA-33829 (3.640 \pm 90) debe ser rechazada también por su bajo $\% \mathrm{C}$ : 0,7 y por haber tratado la muestra solo con ácido (Maroto et al. 2012: 21). Según Jordá et al. (2008: 43) la estimación C14 AA-29664 (12.050 \pm 130$)$ "no cumple los requisitos de orden arqueológico necesarios pues carece de asociación y sincronía con los materiales del nivel datado", por lo que debe ser asimismo descartada. Las tres dataciones obtenidas en el ORAU (19.300 \pm $100,19.310 \pm 80$ y $20.810 \pm 110$ BP) cumplen todos los requisitos exigidos para ser aceptadas: coherencia interna, parámetros químicos en los rangos propuestos en la bibliografía y haber sido tratadas mediante Ultrafiltración (Maroto et al. 2012). Sin embargo son tan sorprendentemente recientes que Baena et al. (2012) no las contemplan en su modelo cronológico. Hay que considerar que se fecharon huesos, cuya acumulación en este nivel se debe principalmente a aportes de carnívoros (Yravedra 2006) que, quizás, los enterraran. Además las características estratigráficas de la unidad favorecen los desplazamientos verticales ("niveles ricos en clastos autóctonos con escasa matriz arenoso lutítica", Jordá et al. 2008: 36). Puede que las dataciones obtenidas en el ORAU estén fechando las actividades de esos animales y no las últimas ocupaciones musterienses del yacimiento. A nuestro juicio, el único nivel musteriense bien datado del tramo superior, ESQ-B, es el VI, cuya edad absoluta/numérica fijamos en $\sim 43 / 44.000$ años BP (estimaciones C14 del ORAU) antes que en 34.380 como defienden Baena et al. (2012). Las otras dataciones de este tramo no deberían ser utilizadas para sustentar la hipótesis de una perduración musteriense/neandertal en la región cantábrica hasta $c a .23 .000$ años BP, ya que o han sido tratadas mediante una secuencia de ácido, o sus parámetros químicos son anómalos, o su asociación con el evento que se pretende datar es incierta.

En resumen, los yacimientos analizados en este artículo tienen serios problemas estratigráficos, tecno-tipológicos y/o cronológicos que cuestionan la interpretación crono-cultural (pervivencia del Musteriense en el norte y sur de la PI) y/o antropológica (hibridación) propuesta en cada caso. Ninguno cuenta con evidencias cronoestratigráficas suficientes que justifiquen una pervivencia neandertal-musteriense en la PI posterior al 40/45.000 BP (sin calibrar). Además, si los grupos musterienses, chatelperronienses y auriñacienses hubiesen 'convivido-coexistido' durante tantos miles de años como se defiende (Baena et al. 2005; Baena et al. 2006; Finlayson et al. 2006; Finlayson et al. 2008; Baena et al. 2012), o si estos complejos no fueran más que facies económicas de un tecnocomplejo mayor (Cabrera et al. 2005; Bernaldo de Quirós et al. 2008), las interestratificaciones mustero-chatelperro-auriñacienses serían una realidad bien documentada estratigráficamente y bien acotada 
cronológicamente, al menos en algunas zonas de la PI como la región cantábrica. Pero nada más lejos de la realidad. Las únicas interestratificaciones constatadas hasta la fecha en la PI ocurren entre el Chatelperroniense y el Auriñaciense en El Pendo (Cantabria) (González Echegaray 1980) y La Güelga (Asturias) (Menéndez et al. 2005, 2006; Menéndez et al. 2009). La primera fue rebatida en los años 1980 por Hoyos y Laville (1982), demostrando la posición secundaria del registro arqueológico y la correlación estratigráfica de los niveles auriñacienses (corte 1) y musterienses (corte 2) (también Barandiarán et al. 1996: 274-275). Sanguino y Morcillo (2001: 75-89) confirmaron después estas críticas. La segunda interestratificación (La Güelga, zona $\mathrm{D}$ interior) se ha identificado en una zona del yacimiento con serios problemas estratigráficos que afectan a los niveles "chatelperronienses" y "auriñacienses" (cono de deyección, discontinuidades erosivas, solifluxiones, derrumbes de visera, madrigueras) (Quesada y Menéndez 2009) y cronológicos (inversión cronológica de las fechas), y con una industria lítica y ósea que nunca supera los 20 artefactos (Menéndez et al. 2005; Menéndez et al. 2006). En este contexto, cualquier tentativa de adscripción cultural resulta cuanto menos aventurada (3). En resumen, las únicas evidencias estratigráficas que respaldan una convivencia-contemporaneidad chatelperro-auriñaciense en la PI carecen de garantías estratigráficas suficientes para sustentar esa hipótesis.

\section{CONCLUSIÓN}

Las técnicas de pretratamiento desarrolladas en la última década en algunos laboratorios de datación como la Ultrafiltración, la Ninhydrina, o el ABOx (Bird et al. 1999; Tisnerat-Laborde et al. 2003; Bronk Ramsey et al. 2004a; Bronk Ramsey et al. 2004b; Higham et al. 2006; Brock et al. 2007; Higham et al. 2009) eliminan de un modo más eficiente los restos de contaminación de las muestras arqueológicas cuyos efectos en la edad absoluta/numérica de las muestras han

(3) Rasilla y Santamaría, 2013. sido señalados en numerosas ocasiones (Schwarcz 1993; Fortea 1995; Fortea et al. 2008; Jöris y Street 2008; Higham 2011). Las dataciones obtenidas con estas técnicas parecen estabilizar la edad del primer auriñaciense en torno a $36 / 37.000$ años BP, a la par que envejecen en algunos miles de años el "Musteriense tardío". Al menos eso se percibe en La Viña (Santamaría 2012), El Sidrón (Torres et al. 2010; Wood et al. 2013), Els Ermitons (Maroto et al. 2012), Fumane (Higham et al. 2009) o Mezmaiskaya (Pinhasi et al. 2011). Es posible que a medida que estas técnicas se depuren y se consigan controlar de un modo más eficaz los efectos de la contaminación, las diferencias entre el Musteriense y el Auriñaciense se incrementen, corroborando por otra parte su posición relativa en la secuencia estratigráfica (i.e. la cronología relativa). Baste recordar a) que los límites aceptados para el inicio y fin de la transición ( $c a$. 40/30.000 años BP) actúan en la práctica como un muro de contención que atenúa los efectos de la contaminación; b) que el porcentaje de contaminación necesario para que una muestra $>45.000$ años BP se date en ca. 25.000 años BP es inferior al 5\%, y que ese porcentaje es perfectamente asumible en muchos yacimientos musterienses de la PI (L'Arbreda, Bajondillo, Gorham's Cave, El Sidrón, Els Ermitons, La Güelga...) y c) que, en general, se desconoce la integridad estratigráfica y cultural de los niveles datados y, sobre todo, que faltan ejemplos claros de interestratificaciones musterochatelperro-auriñacienses.

En este contexto, consideramos que el escenario más plausible, desde un punto de vista cronoestratigráfico, o relativo, es la desaparición del $H$. neanderthalensis/Musteriense antes de la llegada del H. sapiens a la PI. Cabe preguntarse, por fin, si la contaminación de las muestras arqueológicas con carbono moderno es un fenómeno habitual en los yacimientos paleolíticos datados en la transición PM-PS, o por el contrario es un fenómeno accidental y suficientemente controlado. No cabe duda que la contaminación tiene efectos directos en nuestra percepción del pasado, y que a día de hoy carecemos de instrumentos de datación absoluta/numérica lo bastante fiables y precisos para resolver los problemas planteados en el debate: poblamiento, desarrollo interno, sustitución, continuidad, convivencia o intercambios (Fortea 1995; Fortea et al. 2008).

Trab. Prehist., 70, N. ${ }^{\circ}$ 2, julio-diciembre 2013, pp. 241-263, ISSN: 0082-5638

doi: $10.3989 /$ tp.2013.12111 


\section{AGRADECIMIENTOS}

A los Drs. Emilio Aura Tortosa (Universidad de Valencia) y Lourdes Montes Ramírez (Universidad de Zaragoza) la revisión y los comentarios del texto inicial. Este trabajo forma parte del "Programa Severo Ochoa”, FICYT 2006-2009.

\section{BIBLIOGRAFÍA}

Adán, G. y Arsuaga, J. L. 2007: "Nuevas investigaciones en la cueva del Conde (Santo Adriano, Tunón): campañas 2001-2002”. Excavaciones arqueológicas en Asturias (1999-2002). Gobierno del Principado de Asturias. Oviedo: 17-22.

Adler, D. S. y Jöris, O. 2008: "Dating the Middle to Upper Palaeolithic boundary across Eurasia”. Eurasian Prehistory 5 (2): 5-18.

Ambrose, S. H. 1990: "Preparation and characterization of bone and tooth collagen for isotopic Analysis". Journal of archaeological science 17: 431-451.

Andrés, M. 2009: "El Chatelperroniense en la región cantábrica. Estado de la cuestión". Munibe 60: 3550 .

Arbizu, M.; Arsuaga, J. L. y Adán, G. 2005a: “La cueva del Forno/Conde (Tuñón, Asturias): un yacimiento del tránsito del Paleolítico medio y superior en la Cornisa Cantábrica". En R. Montes y J. A. Lasheras (eds.): Neandertales Cantábricos. Estado de la Cuestión. Monografías del Centro de Investigación y Museo de Altamira 20. Ministerio de Cultura. Santander: 423-441.

Arbizu, M.; Arsuaga, J. L. y Adán, G. 2009: “La cueva del Conde 2003-2006 (Proyecto CN 04-218): Neandertales y cromañones en el valle de Tuñón (Santo Adriano)". Excavaciones Arqueológicas en Asturias (2003-2006). Gobierno del Principado de Asturias. Oviedo: 435-446.

Arbizu, M.; Arsuaga, J. L.; Adán, G.; Aramburu, A.; Ellwood, B.; Fombella, M. A.; Álvarez-Lao, D.; García-Menéndez, M. y Fernández-Fernández, J. 2005b: "Las condiciones ambientales durante la transición del Paleolítico medio al superior en la Cornisa Cantábrica: del 40.000 al 30.000 BP en la cueva del Conde (Tuñón, Asturias, España)". En J. Rodríguez-Vidal, C. Finlayson y F. Giles Pacheco (eds.): Cuaternario Mediterráneo y poblamiento de homínidos. Gibraltar Museum y AEQUA. Málaga: 31-32.

Baena, J.; Cabrera, V. y Carrión, E. 2004: "Las sociedades neandertales durante el final del Paleolítico medio". Kobie 8: 91-140.

Baena, J. y Carrión, E. 2006. "Problemas acerca del final del Musteriense”. Zephyrus 59: 51-66.
Baena, J.; Carrión, E.; Cuartero, F. y Fluck, H. 2012: "A chronicle of crisis: The Late Mousterian in north Iberia (Cueva del Esquilleu, Cantabria, Spain)". Quaternary International 247: 199-211.

Baena, J.; Carrión, E.; Ruiz, B.; Ellwood, B.; Sesé, C.; Yravedra, J.; Jordá, J. F.; Uzquiano, P.; Velázquez, R.; Manzano, I.; Sánchez-Marco, A. y Hernández F. 2005: "Paleoecología y comportamiento humano durante el Pleistoceno Superior en la comarca de Liébana: la secuencia de la Cueva de El Esquilleu (Occidente de Cantabria, España)". En R. Montes Barquín y J. A. Lasheras (eds.): Neandertales Cantábricos, estado de la cuestión. Monografías del Museo Nacional y Centro de Investigación de Altamira 20, Ministerio de Cultura. Santander: 461- 487.

Baena, J.; Carrión, E. y Velázquez, R. 2006: "Tradición y coyuntura: claves sobre la variabilidad del musteriense occidental a partir de la cueva del Esquilleu". En V. Cabrera, F. Bernaldo de Quirós y J. M. Maíllo (eds.): En el centenario de la cueva de El Castillo: el ocaso de los Neandertales. Universidad Nacional de Educación a Distancia. Santander: 249-267.

Barandiarán, I.; Fortea, J. y Hoyos, M. 1996: "El Auriñaciense tardío y los orígenes del Gravetiense: el caso de la Región Cantábrica". En A. Montet-White y A. Palma di Cesnola (eds.): XIII International Congress Union International Sciences Préhistoriques et Protohistoriques Coll. XI-XII (Forli 1996): 263-293. Forli.

Barton, C. 1988: Lithic Variability and Middle Paleolithic Behavior. British Archaeological Reports, International Series 408. Archaeopress. Oxford.

Barton, R. 2000: "Mousterian hearths and sellfish: Late neanderthal activities on Gibraltar". En C. Stringer, R. Barton y C. Finlayson (eds.): Neanderthals on the edge. Osbow books. Oxford: 211-220.

Barton, R.; Currant, A.; Fernandez Jalvo, Y.; Finlayson, C.; Goldberg, P.; Macphail, R.; Pettitt, P. y Stringer, C. 1999: "Gibraltar Neanderthals and results of recent excavations in Gorham's, Vanguard and Ibex Caves". Antiquity, 73: 13-23.

Bernaldo de Quirós, F.; Arrizabalaga, A.; Maíllo, J. y Iriarte, M. 2008: "La transición paleolítica mediosuperior en la región centro-oriental de la Cordillera Cantábrica". Espacio, Tiempo y Forma I 1: 33-46.

Bird, M.I.; Ayliffe, L. K.; Fifield, L. K.; Turney, C. S. M.; Cresswell, R. G.; Barrows, T. T. y David, B. 1999: "Radiocarbon dating of 'old' charcoal using a wet oxidation stepped combustion procedure". Radiocarbon 41 (2): 127-140.

Blockley, S. P. F; Bronk Ramsey, C. y Higham, T. 2008: "The Middle to Upper Paleolithic transition: dating, stratigraphy, and isochronous markers". Journal of Human Evolution 55: 764-771.

Braadbaart, F.; Poole, I. y van Brussel, A. A. 2009: "Preservation potential of charcoal in alkaline environments: an experimental approach and implications for the archaeological record". Journal of Archaeological Science 36: 1672-1679. 
Brock, F. y Higham, T. 2009: "AMS Radiocarbon Dating of Paleolithic-Aged Charcoal from Europe and the Mediterranean Rim Using ABOx-SC'. Radiocarbon 51 (2): 839-846.

Brock, F.; Bronk Ramsey, C. y Higham, T. 2007: “Quality assurance of ultrafiltered bone dating". Radiocarbon 49 (2): 187-192.

Bronk Ramsey, C.; Higham, T.; Bowles A. y Hedges, R. 2004a: "Improvements to the pretreatment of bone at Oxford". Radiocarbon 46 (1): 155-163.

Bronk Ramsey, C.; Higham, T. y Leach, P. 2004b: "Towards high-precision AMS: progress and Limitations". Radiocarbon 46 (1): 17-24.

Cabrera, V.; Bernaldo de Quirós, F.; Maíllo, J. M.; Pike,

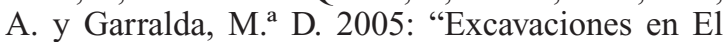
Castillo: veinte años de reflexiones". En R. Montes y J. A. Lasheras (eds.): Neandertales Cantábricos, Estado de la Cuestión. Monografías del Museo Nacional y Centro de Investigación de Altamira 20, Ministerio de Cultura. Santander: 505-526.

Cabrera, V.; Hoyos, M. y Bernaldo de Quirós, F. 1993: "La transición del Paleolítico Medio al Superior en la cueva de El Castillo: características paleoclimáticas y situación cronológica". En V. Cabrera (ed.): El origen del Hombre moderno en el suroeste de Europa. Universidad Nacional de Educación a Distancia. Madrid: 81-101.

Cabrera, V.; Maíllo, J. M. ; Lloret, M. y Bernaldo de Quirós, F. 2001: "La transition vers le Paléolithique Supérieur dans la grotte du El Castillo (Cantabrie, Espagna): la couche 18'. L'Anthropologie 105: 505532.

Chappell, J.; Head, J. y Magee, J. 1996: "Beyond the radiocarbon limit in Australian archaeology and Quaternary research". Antiquity 70: 543-552.

DeNiro, M. J. y Weiner, S. 1988: “Chemical, enzymatic and spectroscopic characterization of 'collagen' and other organic fractions from prehistoric bones". Geochimica et Cosmochimica Acta 52: 2197-2206.

Finlayson, C.; Fa, D. A.; Jiménez Espejo, F.; Carrión, J. S.; Finlayson, G.; Giles Pacheco, F.; Rodríguez Vidal, J.; Stringer, C. y Martínez Ruiz, F. 2008: "Gorham's Cave, Gibraltar, The persistence of a Neanderthal population". Quaternary International 181: 64-71.

Finlayson, C.; Giles Pacheco, F.; Rodriguez-Vidal, J.; Fa, D. A.; Gutierrez Lopez, J.; Santiago Perez, A.; Finlayson, G.; Allue, E.; Baena, J.; Caceres, I.; Carrion, J. S.; Fernandez Jalvo, Y.; Gleed-Owen, C. P.; Jimenez Espejo, F. J.; Lopez, P.; Lopez Saez, J. A.; Riquelme Cantal, J.A.; Sanchez Marco, A.; Giles, F.; Brown, K.; Fuentes, N.; Valarino, C. A.; Villalpando, A.; Stringer, C.; Martinez Ruiz, F. y Sakamoto, T. 2006: "Late survival of Neanderthals at the southernmost extreme of Europe". Nature 443: 850-853. "Supplementary information. Late survival of Neanderthals at the southernmost extreme of Europe". doi:10.1038/nature05195.
Fortea, J. 1995: "Abrigo de La Viña. Informe y primera valoración de las campañas 1991 a 1994". Excavaciones Arqueológicas en Asturias (1991-94). Principado de Asturias. Oviedo: 19-31.

Fortea, J.; Rasilla, M.; Santamaría, D. y Rosas, A. 2008: "El Paleolítico superior antiguo en Asturias y su contexto". En J. Rodríguez Muñoz (ed.): La Prehistoria en Asturias. Un legado artístico único en el mundo. La Nueva España. Oviedo: 355-388.

García, M.; Adán, G.; Arsuaga, J. L.; Arbizu, M. y Alvárez-Laó, D. 2006: "L'industrie lithique de passage du Paléolithique moyen au supérieur de la grotte de El Conde: premières donnés". Book of abstracts. $X V_{\text {th }}$ Congress of the International Union for Prehistoric and Protohistoric Sciences (Lisboa 4-9 September) 2: 709-710.

Gibert, J. y Walker, M. J. 1999: "La Sima de las Palomas del Cabezo Gordo (Dolores de Pacheco, Torre Pacheco, Murcia): Los trabajos realizados en 1994". Memorias de arqueología 9, Comunidad Autónoma de Murcia. Murcia: 11-17.

Giles Pacheco, F.; Giles Guzmán, F. J.; Gutiérrez, J. M.; Pérez, A. S.; Finlayson, C.; Rodríguez Vidal, J.; Finlayson, G. y Fa, D. A. 2012: "The tools of the last Neanderthals: Morphotechnical characterization of the lithic industry at level IV of Gorham's Cave. Gibraltar". Quaternary International 247: 151-161.

Gillespie R. 1997: "Burnt and unburnt carbon: dating charcoal and burnt bone from the Willandra Lakes, Australia". Radiocarbon 39 (3): 225-36.

Godwin H. 1962: "Half-life of radiocarbon". Nature 195: 984.

González Echegaray, J. 1980: El yacimiento de la cueva de "El Pendo" (Excavaciones 1953-57). Bibliotheca Praehistorica Hispana XVII, CSIC. Madrid.

Hedges, R. E. M. y van Klinken, G. M. 1992: “A review of current approaches in the pretreatment of bone for radiocarbon dating by AMS". Radiocarbon 34 (3): 279-291.

Higham, T. 2011: "European Middle and Upper Palaeolithic radiocarbon dates are often older than they look: problems with previous dates and some remedies". Antiquity 85: 235-249.

Higham, T.; Brock, F.; Peresani, M.; Broglio, A.; Wood, R. y Douka, K. 2009: "Problems with radiocarbon dating the Middle to Upper Palaeolithic transition in Italy". Quaternary Science Reviews 28: 1257-1267.

Higham, T.; Jacobi, R.y Bronk Ramsey, C. 2006: "AMS radiocarbon dating of ancient bone using Ultrafiltration". Radiocarbon 48: 179-195.

Hoyos, M. y Laville, H. 1982: "Nuevas aportaciones sobre la estratigrafía y sedimentología de los depósitos del Paleolítico Superior de la Cueva de El Pendo (Santander): sus implicaciones". Zephyrus $X X X I V-X X X V: 285-295$.

Hublin, J. J.; Barroso, C.; Medina, P.; Fontugne, M. y Reyss, J.L. 1995: "The Mousterian site of Zafarraya (Andalucia, Spain): dating and implications on the

Trab. Prehist., 70, N. ${ }^{\circ}$ 2, julio-diciembre 2013, pp. 241-263, ISSN: 0082-5638

doi: $10.3989 /$ tp.2013.12111 
palaeolithic peopling processes of Western Europe". Compte Rendus de l'Académie des Sciences de Paris 321 serie IIa: 931-937.

Jacobi, R. M.; Higham, T. y Bronk Ramsey, C. 2006: "AMS radiocarbon dating of Middle and Upper Palaeolithic bone in the British Isles: improved reliability using ultrafiltration". Journal of Quaternary Science 21: 557-573.

Jennings, R.; Giles Pacheco, F.; Barton, R.; Collcut, S.; Gale, R.; Gleed-Owen, C. P.; Gutiérrez López, J.; Higham, T. F. G.; Parker, A.; Price, C.; Rhodes, E.; Santiago Perez, A.; Schwenninger, J. y Turner, E. 2009: "New dates and palaeoenvironmental evidence for the Middle to Upper Palaeolithic occupation of Higueral de Valleja Cave, southern Spain". Quaternary Science Reviews 28: 830-839.

Jordá, J. F.; Baena, J.; Carral, P.; García-Guinea, J.; Correcher, V. e Yravedra, J. 2008: "Procesos sedimentarios y diagenéticos en el registro arqueológico del yacimiento pleistoceno de la cueva de el Esquilleu (Picos de Europa, Norte de España)". Revista Cuaternario y Geomorfología 22 (3-4): 31-46.

Jöris, O.; Álvarez, E. y Weninger, B. 2003: "Radiocarbon evidence of the Middle to Upper Palaeolithic transition in southwestern Europe". Trabajos de Prehistoria 60 (2): 15-38.

Jöris, O. y Street, M. 2008: "At the end of C14 time scale -the Middle to Upper Paleolithic record ofwestern Eurasia". Journal of Human Evolution 55: 782-802.

Jöris, O.; Street, M.; Terberger, T. y Weninger, B. 2011: "Radiocarbon dating the Middle to Upper Palaeolithic transition: The demise of the last Neanderthals and the first appearance of anatomically modern humans in Europe". En S. Condemi y G. C. Weniger (eds.): Continuity and discontinuity in the peopling of Europe: one hundred fifty years of Neanderthal study. Springer Netherlands: 239-298.

Laville, H. y Hoyos, M. 1994: "Algunas precisiones sobre la estratigrafía y sedimentología de Cueva Morín (Santander)". En F. Bernaldo de Quirós (ed.): El cuadro geocronológico del Paleolítico Superior Inicial. Monografías del Museo Nacional y Centro de Investigación de Altamira 13, Ministerio de Cultura. Madrid: 199-209.

López-García, J. M.; Cuenca-Bescós, G.; Blain, H. A.; Álvarez-Lao, D.; Uzquiano, P.; Adán, G.; Arbizu, M. y Arsuaga, J. L. 2011: "Palaeoenvironment and palaeoclimate of the Mousterian- Aurignacian transition in northern Iberia: The small-vertebrate assemblage from Cueva del Conde (Santo Adriano, Asturias)". Journal of Human Evolution 61: 108-116.

Maíllo, J. M. 2002: "Tecnología lítica en el Auriñaciense arcaico de Cueva Morín (Villanueva de Villaescusa, Cantabría)". Espacio, Tiempo y Forma I 15: 87-116.

Maíllo, J. M. 2005a: "Esquemas operativos líticos del Musteriense Final de Cueva Morín (Villanueva de Villaescusa, Cantabria)". En R. Montes y J. A. Lasheras (eds.): Neandertales Cantábricos, estado de la cuestión. Monografías del Museo Nacional y Centro de Investigación de Altamira 20, Ministerio de Cultura. Santander: 301-313.

Maíllo, J. M. 2005b: "La producción laminar en el Chatelperroniense de Cueva Morín: modalidades, intenciones y objetivos". Trabajos de Prehistoria 62 (1): 47-64.

Maíllo, J. M. 2007: "Le Châtelperronien en Espagne: mythes et réalités. Une approche Technologique". En J. Evin (ed.): Congrès du Centenaire. Un siècle de construction du discours scientifique en Préhistoire. Mémoires de la Société Préhistorique Française. Paris: 95-103.

Maroto, J.; Vaquero, M.; Arrizabalaga, A.; Baena, J.; Baquedano, E.; Jordá, J.; Julià, R.; Montes, R.; Van der Plicht, J.; Rasines, P. y Wood, R. 2012: "Current issues in late Middle Palaeolithic chronology: New assessments from northern Iberia". Quaternary International 247: 15-25.

Menéndez, M.; García, E. y Quesada, J. M. 2005: "La transición Paleolítico Medio-Paleolítico Superior en la Cueva de La Güelga (Cangas de Onís, Asturias). Un avance a su registro". En R. Montes y J. A. Lasheras (eds.): Neandertales Cantábricos. Estado de la Cuestión. Monografías del Centro de Investigación y Museo de Altamira, 20. Ministerio de Cultura. Madrid: 589-617.

Menéndez, M.; García, E. y Quesada, J. M. 2006: "Excavaciones en la cueva de la Güelga (Cangas de Onís, Asturias)". En V. Cabrera, F. Bernaldo de Quirós y J. M. Má́llo (eds.): En el centenario de la cueva de El Castillo: el ocaso de los neandertales. Universidad Nacional Educación a Distancia. Santoña: 209-229.

Menéndez, M.; Quesada, J. M.; Jordá, J. F.; Carral, P.; Trancho, G.; García, E.; Álvarez, D.; Rojo, J. y Wood, R. 2009: "Excavaciones arqueológicas en la cueva de la Güelga (Cangas de Onís)". Excavaciones arqueológicas en Asturias (2003-2006). Gobierno del Principado de Asturias. Oviedo: 209-221.

Mercier, N. y Valladas, H. 1993: "Contribution des méthodes de datation par le carbone 14 et la thermoluminescence à la chronologie de la transition du Paléolithique moyen au Paléolithique supérieur". En V. Cabrera (ed.): El origen del hombre moderno en el suroeste de Europa. Universidad Nacional Educación a Distancia. Madrid: 47-60.

Mook, W. G. 2002: Isótopos ambientales en el ciclo hidrológico: principios y aplicaciones. Instituto Geológico y Minero de España. Madrid.

Pessenda, L.; Gouveia, S. y Aravena, R. 2001: "Radiocarbon dating of total soil organic matter and humin fraction and its comparison with $14 \mathrm{C}$ ages of fossil charcoal". Radiocarbon 43(2): 595-601.

Pettitt, P. y Bailey, R. 2000: "AMS Radiocarbon and Luminescence Dating of Gorham's and Vanguard 
Caves, Gibraltar, and Implications for the Middle to Upper Paleolithic Transition in Iberia". En C. Stringer, R. Barton y C. Finlayson (eds.): Neanderthals on the edge. Osbow books. Oxford: 155-162.

Pettitt, P. y Pike, A. 2001: "Blind in a Cloud of Data. Problems with the Chronology of Neanderthal Extinction and Anatomically Modern Human Expansion". Antiquity 75: 415-420.

Pinhasi, R.; Higham, T.; Golovanova, L. V. y Doronichev, V. B. 2011: "Revised age of late Neanderthal occupation and the end of the Middle Paleolithic in the northern Caucasus". Proceedings of the National Academy of Sciences 108, 21: 8611-8616.

Quesada, J. M. y Menéndez, M. 2009: “Revisión cronoestratigráfica de la cueva de la Güelga (Narciandi, Asturias). Del Musteriense al Paleolítico superior inicial". Espacio, Tiempo y Forma I 2: 39-74.

Rasilla, M. de la y Santamaría, D. 2013: "El Paleolítico medio en Asturias". Mainake XXXIII: 37-68.

Reimer, P. J.; Baillie, M. G. L.; Bard, E; Bayliss, A; Beck, J. W.; Blackwell, P. G.; Bronk Ramsey, C; Buck, C. E.; Burr, G. S.; Edwards, R. L.; Friedrich, M; Grootes, P. M.; Guilderson, T. P.; Hajdas, I; Heaton, T. J.; Hogg, A. G.; Hughen, K. A.; Kaiser, K. F.; Kromer, B; McCormac, F. G.; Manning, S. W.; Reimer, R. W.; Richards, D. A.; Southon, J. R.; Talamo, S.; Turney, C. S. M.; van der Plicht, J.; Weyhenmeyer, C. E. 2009: "IntCal09 and Marine09 Radiocarbon Age Calibration Curves, 0-50,000 Years cal BP". Radiocarbon 51: 1111-1150.

Sanguino, J. y Morcillo, C. 2001: "La secuencia paleolítica clásica. Descripción, problemática y reinterpretación estratigráfica”. En R. Montes Barquín y J. Sanguino González (eds.): La cueva de El Pendo. Actuaciones arqueológicas 1994-2000. Consejería de Cultura, Educación y Deporte. Santander: 75-89.

Santamaría, D. 2012: La transición del Paleolítico medio al superior en Asturias. El Abrigo de La Viña (La Manzaneda, Oviedo) y la Cueva de El Sidrón (Borines, Piloña). Servicio de Publicaciones de la Universidad de Oviedo. Oviedo.

Santamaría, D.; Fortea, J.; Rasilla, M. de la; Martínez, L.; Martínez, E.; Cañaveras, J.; Sánchez-Moral, S.; Rosas, A.; Estalrrich, A.; García-Tabernero, A. y Lalueza-Fox, C. 2010: "The technological and typological behaviour of a Neanderthal group from El Sidrón cave (Asturias, Spain)". Oxford Journal of Archaeology 29 (2): 119-148.

Santamaría, D.; Rasilla, M. de la; Martínez, L. y Tarriño, A. 2011: "Las herramientas y su interpretación cultural y económica". En M. de la Rasilla, A. Rosas, J. C. Cañaveras y C. Lalueza-Fox (eds.): La cueva de El Sidrón (Borines, Piloña, Asturias) Investigación interdisciplinar de un grupo neandertal. Excavaciones Arqueológicas en Asturias, Monografías I, Principado de Asturias. Oviedo: 137-146.

Schoeninger, M. J.; Moore, K. M.; Murray, M. L. y Kingston, J. D. 1989: "Detection of bone preserva- tion in archaeological and fossil samples". Applied Geochemistry 4: 281-292.

Schwarcz, H. 1993: "Problems and limitations of absolute dating of the appearance of modern man in southwestern Europe". En V. Cabrera (ed.): El origen del hombre moderno en el suroeste de Europa. Universidad Nacional de Educación a Distancia. Madrid: 23-45.

Stuiver M. y Polach H. 1977: "Discussion: Reporting of 14C data". Radiocarbon 19 (3): 355-363.

Tisnerat-Laborde, N.; Valladas, H.; Kaltnecker, E. y Arnold, M. 2003: "AMS radiocarbon dating of bones at LSCE". Radiocarbon 45: 409-419.

Torres, T de.; Ortiz, J.; Grün, R.; Eggins, S.; Valladas, H.; Mercier, N.; Tisnérat-Laborde, N.; Juliá, R.; Soler, V.; Martínez, E.; Sánchez-Moral, S.; Cañaveras, J.; Lario, J.; Badal, E.; Lalueza-Fox, C.; Rosas, A.; Santamaría, D.; Rasilla, M. de la y Fortea, J. 2010: "Dating of the hominid (Homo neanderthalensis) remains accumulation from el Sidrón cave (Piloña, Asturias, north Spain): an example of a multimethodological approach to the dating of upper Pleistocene sites". Archaeometry 52 (4): 680-705.

Torres, T de.; Ortiz, J.; Grün, R.; Eggins, S.; Valladas, H.; Mercier, N.; Tisnérat-Laborde, N.; Juliá, R.; Soler, V.; Santamaría, D. y Rasilla, M. de la. 2011: "La datación de los fósiles de El Sidrón: una compleja aproximación multidisciplinar". En M. de la Rasilla, A. Rosas, J. C., Cañaveras y C. Lalueza (eds.): La cueva de El Sidrón (Borines, Piloña, Asturias). Investigación interdisciplinar de un grupo neandertal. Excavaciones Arqueológicas en Asturias, Monografías I, Principado de Asturias. Oviedo: 159-165.

Van Klinken, G. J. 1999: "Bone Collagen Quality Indicators for Palaeodietary and Radiocarbon Measurements". Journal of Archaeological Science 26: 687-695.

Walker, M. J. y Gibert, J. 2002a: La Sima de las Palomas del Cabezo Gordo en Dolores de Pacheco de Torre Pacheco: campaña 1995. Memorias de Arqueología 10, Región de Murcia. Murcia: 11-19.

Walker, M. J. y Gibert, J. 2002b: La Sima de las Palomas del Cabezo Gordo en Dolores de Pacheco de Torre Pacheco: campaña 1996. Memorias de Arqueología 11, Región de Murcia. Murcia: 19-33.

Walker, M. J. y Gibert, J. 2004: La Sima de las Palomas del Cabezo Gordo en Dolores de Pacheco de Torre Pacheco: campaña 1997. Memorias de Arqueología 12, Región de Murcia. Murcia: 37-44.

Walker, M. J. y Gibert, J. 2005: La Sima de las Palomas del Cabezo Gordo en Dolores de Pacheco de Torre Pacheco: campaña 1998. Memorias de Arqueología 13, Región de Murcia. Murcia: 11-18.

Walker, M. J.; Gibert, J.; López, M. V.; Lombardi, A.V.; Pérez, A.; Zapata, J.; Ortega, J.; Higham, T.; Pike, A.; Schwenninger, J. L.; Zilhão, J. y Trinkaus, E. 2008: "Late Neandertals in Southeastern Iberia: Sima de las Palomas del Cabezo Gordo, Murcia,

Trab. Prehist., 70, N. ${ }^{\circ}$ 2, julio-diciembre 2013, pp. 241-263, ISSN: 0082-5638

doi: $10.3989 /$ tp.2013.12111 
Spain". Proceedings of the National Academy of Sciences 105 (52): 20631-20636. "Supporting information. Late Neandertals in Southeastern Iberia: Sima de las Palomas del Cabezo Gordo, Murcia, Spain". doi: 10.1073/pnas.0811213106

Walker, M. J.; Gibert, J.; López, M. y López, A. 2006: La Sima de las Palomas del Cabezo Gordo, en Dolores de Pacheco de Torre Pacheco: campaña 1999. Memorias de Arqueología 14, Región de Murcia. Murcia: 63-78.

Weninger, B. y Jöris, O. 2008: "A C14 age calibration curve for the last $60 \mathrm{ka}$ : the Greenland-Hulu U/Th timescale and its impact on understanding the Middle to Upper Paleolithic transition in Western Eurasia”. Journal of Human Evolution 55 (5): 772-81.

Wood, R. E; Higham, T. F. G.; Torres, T de; TisnératLaborde, N.; Valladas, H.; Ortiz, J. E.; Lalueza, C.; Sánchez-Moral, S.; Cañaveras, J. C.; Rosas, A.; Santamaría, D.; Rasilla, M. de la 2013: "A new date for the Neanderthals from El Sidrón Cave (Asturias, Northern Spain)". Archaeometry 55, 1: 148-158.

Yravedra, J. 2006: "Acumulaciones biológicas en yacimientos arqueológicos: Amalda VII y Esquilleu III-IV". Trabajos de Prehistoria 63 (2): 55-78.

Zilhão, J. 1993: "Le passage du Paléolithique moyen au Paléolithique supérieur dans le Portugal". En V.
Cabrera (ed.): El origen del hombre moderno en el suroeste de Europa. Universidad Nacional de Educación a Distancia. Madrid: 127-145.

Zilhão, J. 2000: "The Ebro Frontier: A Model for the Late Extinction of Iberian Neanderthals". En B. Stringer, R. N. Barton y J. C. Finlayson (eds.): Neanderthals on the Edge. Oxbow Books. Oxford: 111-121.

Zilhão, J. 2006: "Chronostratigraphy of the Middle-toUpper Paleolithic Transition in the Iberian Peninsula". Pyrenae 37 (1): 7-84.

Zilhão, J. y d'Errico, F. 1999: "The chronology and taphonomy of the earliest Aurignacian and its implications for the understanding of Neandertal extinction". Journal of World Prehistory 13: 1-68.

Zilhão, J. y d'Errico, F. 2000: "La nouvelle 'bataille aurignacienne"'. L'Anthropologie 104: 17-50.

Zilhão, J. y d'Errico, F. 2003: "The chronology of the Aurignacian and transitional technocomplexes. Where do we stand?'. En J. Zilhão y F. d'Errico (eds.): The chronology of the Aurignacian and of the transitional technocomplexes. Dating, stratigraphies, cultural implications. Actes Congrès UISPP (Lieja, Bélgica 2001). Trabalhos de arqueologia 33, Instituto Portugués de Arqueología. Lisboa: 313-349.

Zilhão, J. y Pettitt, P. 2006: "On the new dates for Gorham's Cave and the late survival of Iberian Neanderthals". Before Farming 3: 1-9. 